\title{
Rare Moss-Built Microterraces in a High-Altitude, Acid Mine Drainage-Polluted Stream (Cordillera Negra, Peru)
}

\author{
Jan Sevink • Jacobus M. Verstraten • Annemieke M. Kooijman • \\ Raul A. Loayza-Muro • Leo Hoitinga • Edwin J. Palomino • Boris Jansen
}

Received: 26 October 2014 / Accepted: 20 March 2015 / Published online: 29 May 2015

(C) The Author(s) 2015. This article is published with open access at Springerlink.com

\begin{abstract}
The Rio Santiago in the Cordillera Negra of Peru is severely contaminated by acid mine drainage in its headwaters. In a strongly acid stream, at about 3800 $\mathrm{m}$ above sea level (masl), microterraces were found with terrace walls built up of dead moss, with encrustations and interstitial fine, creamy sediment. The stream water was turbid due to the presence of similar suspended sediment, which also occurred as a thin basal layer in inter-rim basins. The moss was identified as the rare bryophyte Anomobryum prostratum (Müll. Hal.) Besch. Chemical and mineralogical analyses show that green, living parts of the moss are gradually coated by $\mathrm{Al} / \mathrm{Fe}$ (hydr)oxides, inducing their senescence and death. The necromass is covered by creamy crusts through precipitation of schwertmannite-type material from the stream water and simultaneous 'capture' of fine sediment. The latter consists of a mixture of precipitate and fine detrital primary minerals. These processes are
\end{abstract}

Electronic supplementary material The online version of this article (doi:10.1007/s11270-015-2390-x) contains supplementary material, which is available to authorized users.

J. Sevink $(\bowtie) \cdot$ J. M. Verstraten · A. M. Kooijman •

L. Hoitinga $\cdot$ B. Jansen

Institute for Biodiversity and Ecosystem Dynamics,

Amsterdam, The Netherlands

e-mail: j.sevink@uva.nl

R. A. Loayza-Muro

Laboratory of Ecotoxicology, Universidad Peruana Cayetano

Heredia, Lima 31, Peru

E. J. Palomino

Faculty of Environmental Sciences, Universidad Nacional

'Santiago Antúnez de 31 Mayolo', Huaraz, Peru held responsible for the formation of the microterraces, which regarding their composition and environment seem to be unique. Remarkable is the high As content of the creamy crusts and sediment, attributed to strong sorption of As, whereas its solute concentration is relatively low. This calls for more attention to suspended fine sediment in the assessment of environmental risks of stream water use. Lastly, the results raise serious doubts about the use of aquatic bryophytes as bioindicator for chemical pollution in acid mine drainage-polluted streams.

Keywords Acid mine drainage $\cdot$ Arsenic $\cdot$ Bryophyte . Microterraces $\cdot$ Schwertmannite

\section{Introduction}

The Cordillera Negra in Ancash (Peru) is noted for its polymetallic mines (Walsh 2013). Loayza-Muro et al. (2010) studied the heavy metal pollution and other environmental stress factors for the aquatic entomofauna in its high-altitude streams, inclusive of the Rio Santiago in the Aija catchment (see Fig. 1). During a visit to their Rio Santiago sampling site in November 2010 (end of the dry season), we observed hitherto unnoticed travertine-like microterraces that were built up by a single moss species; the only macro plant species found. Inter-rim basins held cream-coloured fine sediment, also encountered as interstitial fill in the moss rims.

Moss-built terraces are known from highly calcareous environments in which travertine is formed (e.g. 

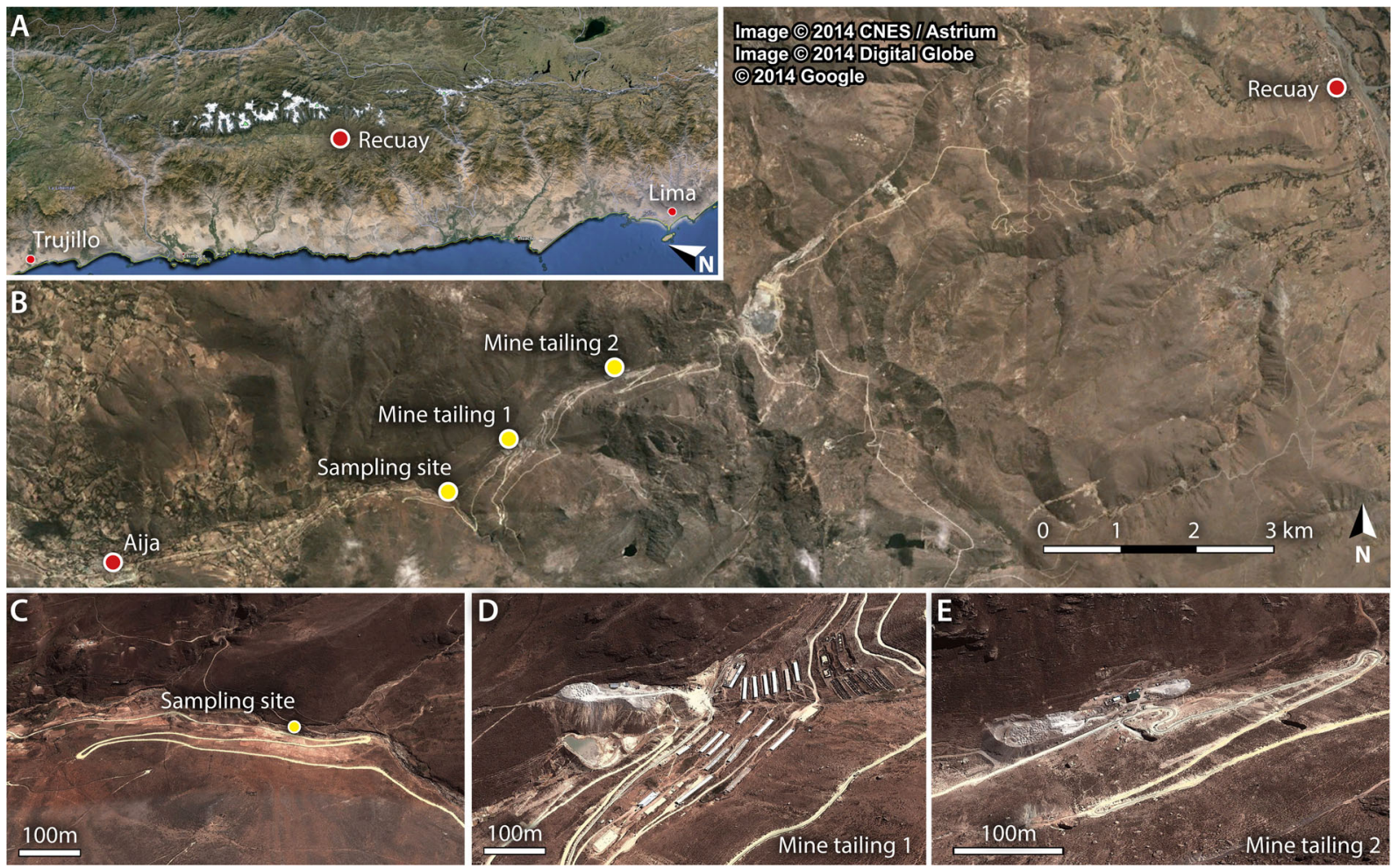

Fig. 1 Location of the area of study (a, b), sampling site (b, c) and major upstream mine tailings (d, e)

Pentacost 2005), but not from such fundamentally different and extreme environments as that of the Rio Santiago: extremely acid, highly polluted, and at about $3800 \mathrm{~m}$ above sea level (masl) (see Table 1). This paper concerns a case study of such unusual aquatic system with emphasis on the composition and origin of the moss-built microterraces and fine suspended sediment, the identification of the moss species, and its survival in this extreme environment.

\section{Environmental Setting}

The catchment is in the central part of the Cordillera Negra, west of Recuay (Fig. 1). Data on its climate are truly scarce. It is a relatively dry tropical puna climate with distinct seasonal precipitation, which probably ranges from 500 to $1000 \mathrm{~mm}$ annually. Loayza-Muro et al. (2010) observed a roughly twofold increase in wet season discharge of the Rio Santiago at 3800 masl, while the water temperature ranged from $11.5^{\circ} \mathrm{C}$ (wet season: December-April) to $9.3{ }^{\circ} \mathrm{C}$ (dry season), suggesting a similar small range in mean monthly air temperatures.

The main geological unit is the Early Tertiary Calipuy Group, composed of varied volcanic strata, but in places strata are non-volcanic and even some limestone beds were observed (Bodenlos and Straczek 1957). To the west, towards Aija, a large intrusive complex occurs, while some minor intrusive bodies have been found with associated polymetallic ore deposits close to the sampling site (MRC1 n.d; Chirif et al. 2010). Ores from several mines in the upper Rio Santiago catchment near the Huancapeti pass are processed in nearby plants, and residues are dumped in huge tailings and reservoirs (see Fig. 1). Waters from these dumps are very acid, $\mathrm{pH}$ values of 3 or even lower being reported by LoayzaMuro et al. (2010) and Walsh (2013).

Studies on the flora of the Cordillera Negra are rare and seemingly inexistent for the high, central part of this range. The few studies concern specific plants, such as Brassica spp. (Monsalve and Cano 2003) and lichens (Ramírez and Cano 2005). Reports on mosses are limited to a few early publications with descriptions of locations at which specific species were found (Zander and Hegewald 1976; Hegewald and Hegewald 1977 and 1985).

Figure 2 shows several aspects of the area around the sampling site, which is at 3800 masl. The riverbed is in the country rock (probably acid igneous rock; Chirif et al. 2010) and partially filled with very coarse textured 
Table 1 Chemical composition of the stream water

\begin{tabular}{|c|c|c|}
\hline & This study & Loayza et al. \\
\hline & With filtration & Without filtration $^{\mathrm{b}}$ \\
\hline $\mathrm{O}_{2}\left(\mathrm{mg} \mathrm{L}^{-1}\right)$ & nd & 5 \\
\hline Temperature $\left({ }^{\circ} \mathrm{C}\right)$ & nd & 11.5 \\
\hline $\mathrm{pH}^{\mathrm{a}}$ & 3.3 & 3.4 \\
\hline \multirow[t]{2}{*}{$\mathrm{EC} 25\left(\text { microS } \mathrm{cm}^{-1}\right)^{\mathrm{a}}$} & 1960 & 1776 \\
\hline & $\mu \mathrm{mol} \mathrm{L}{ }^{-1}$ & $\mu \mathrm{mol} \mathrm{L}{ }^{-1}$ \\
\hline K & 114 & 34 \\
\hline $\mathrm{Na}$ & 573 & 256 \\
\hline $\mathrm{Ca}$ & 4110 & 1491 \\
\hline $\mathrm{Mg}$ & 943 & 704 \\
\hline $\mathrm{Sr}$ & 11.2 & nd \\
\hline $\mathrm{Al}$ & 2.6 & 484 \\
\hline $\mathrm{Fe}$ & 5000 & 671 \\
\hline $\mathrm{Mn}$ & 1055 & 358 \\
\hline $\mathrm{Zn}$ & 1030 & 246 \\
\hline $\mathrm{Pb}$ & $<0.25$ & 4.2 \\
\hline $\mathrm{Cd}$ & 0.4 & 4.5 \\
\hline $\mathrm{Si}$ & 341 & 445 \\
\hline $\mathrm{SO}_{4}$ & 12,210 & nd \\
\hline $\mathrm{Cl}$ & 252 & nd \\
\hline As & 104 & 47 \\
\hline ortho-P & 1.9 & 1.8 \\
\hline $\mathrm{NH}_{4}$ & 415 & 1.8 \\
\hline $\mathrm{NO}_{3}$ & $<3$ & nd \\
\hline
\end{tabular}

Data from Loyaza et al. (2010) for the same stream with $n=4$

$n d$ not determined

${ }^{\text {a }}$ Comparable data

${ }^{\mathrm{b}}$ Acidified with $10 \mathrm{M} \mathrm{HNO}_{3}$

and poorly sorted fluvial deposits. Iron hydroxides thinly coat the bedrock and sediment to the level reached during flood. The top of the microterraces built up by the moss is virtually horizontal. Rims are up to $2 \mathrm{~m}$ long and several decimetres high. Moss fills gaps in between the boulders and blocks, retaining water in small basins that may be several decimetres deep.

The stream is turbid, with very fine cream-coloured suspended material. In the basins, it has accumulated in a bottom layer that is up to several centimetres thick. Particles, when carried with the water over the rims, are partially caught by the moss (Fig. 2) and fill its interstices. General data on the stream are provided by Loayza-Muro et al. (2010): discharge during the dry season is about $50 \mathrm{~L} / \mathrm{s}$, mean temperature is about $10{ }^{\circ} \mathrm{C}(n=4)$, and the oxygen content of the turbulent and thus oxygenated stream is about $5 \mathrm{mg} / \mathrm{L}( \pm 0.74 ; n=4)$.

No indications (e.g. high flood lines in the river bed or physical damage to the terrace rims) were found for exceptional rainstorms or dam breaks that might have led to incidental flushes of polluted water from the mine dumps upstream of the site in the previous period (the dry season), compromising the relevance of our water sampling for the longer-term stream water composition during this dry season, nor were such events reported by interviewed locals.

\section{Materials and Methods}

Samples were taken on the 28th of November 2010, at the end of the dry season. Stream water and creamy material was sampled in small polyethylene bottles $(n=$ 2). Bottles were stored at $4{ }^{\circ} \mathrm{C}$ in Peru and the Netherlands, interrupted by their transport (packed in insulating foam) to the Netherlands. They were filtered over a $0.2-\mu \mathrm{m}$ membrane on the 2 nd of December 2010 (e.g. 5 days later). Filtrates were combined into one water sample that was used for chemical analysis of solutes. After filtration, part of the water sample was acidified with $\mathrm{HNO}_{3}$, the other part remained untreated. Both were analysed within a month. The remaining material was combined into one sediment sample (sample P1), washed and centrifuged, subsequently freeze-dried and used for further analyses. Creamy sediment was resampled in November 2011 and after immediate transport to the Netherlands, washed and centrifuged, freezedried and also used for chemical analysis (sample P2).

To check for changes in stream water composition during transport and storage, $\mathrm{pH}$ and electrical conductivity (EC) values were established immediately after sampling in Peru and again prior to filtration in the lab (within 5 days after sampling). No changes in $\mathrm{pH}, \mathrm{EC}$, and colour of the creamy material were observed, as could be expected considering the short period of storage under appropriate conditions and the turbulent and oxygenated conditions in the stream.

Moss samples were taken from one of the rims, packed in polyethylene bags and insulating foam and kept in a refrigerator at $4{ }^{\circ} \mathrm{C}$ in Peru and the Netherlands. Part was freeze-dried after washing to conserve the plant structure and used for its identification. The vertical stratification in the moss rims was studied by cutting slices from a large block as indicated in Fig. 3, washing 

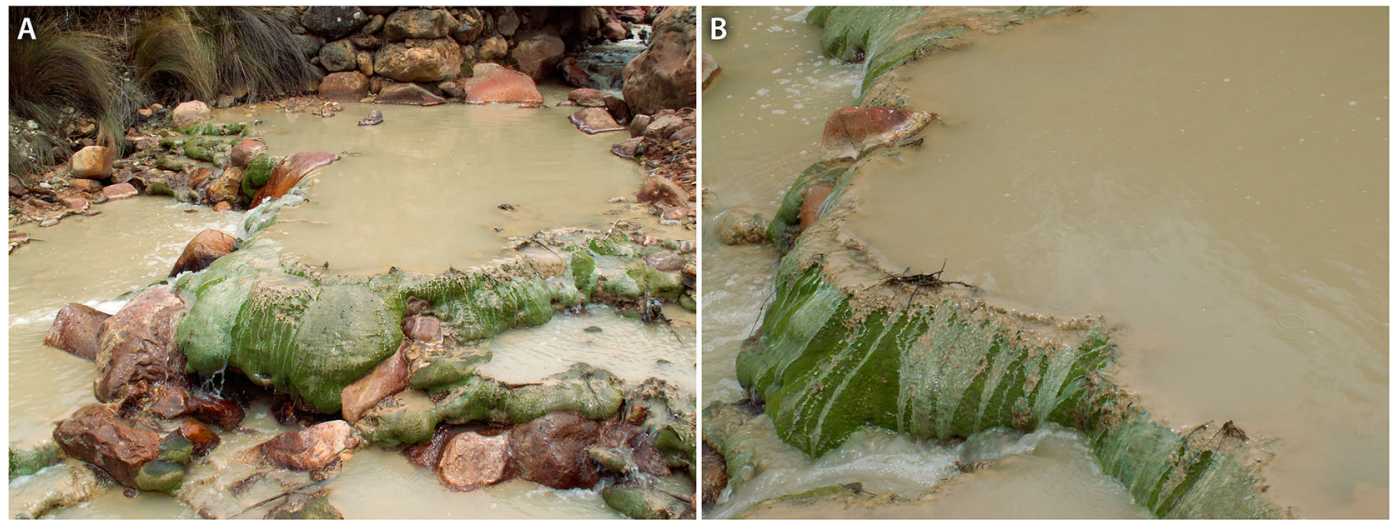

Fig. 2 a The stream with its moss-built terraces and rust-stained coarse debris. b Detail of rim and basin showing the creamy sediment and hydrophobic moss surface

these slices with demi-water over a sieve, followed by centrifugation and freeze-drying of the sediment obtained (P3-P7), and their chemical analysis. Chemical analyses were performed on samples of green, living moss carefully razor cut from the upper $3-4 \mathrm{~mm}$ of a moss monolith (see Figs. 2 and 3) to establish the composition of this plant material.

The creamy material (both suspended and mosscaptured) was microscopically studied, using a Leitz petrographic microscope (magnification up to $\times 500$ ), and both moss and creamy material were studied with a Leitz stereomicroscope at lower magnifications (up to $\times 50$ ).

Chemical analyses were performed on moss and sediment samples using several methods:

- Method 1-after ignition of the dry sample $(n=2)$ to destroy organic matter and destruction in a hot $\mathrm{HF} /$ $\mathrm{H}_{2} \mathrm{SO}_{4}$ mixture, followed by dissolution of the

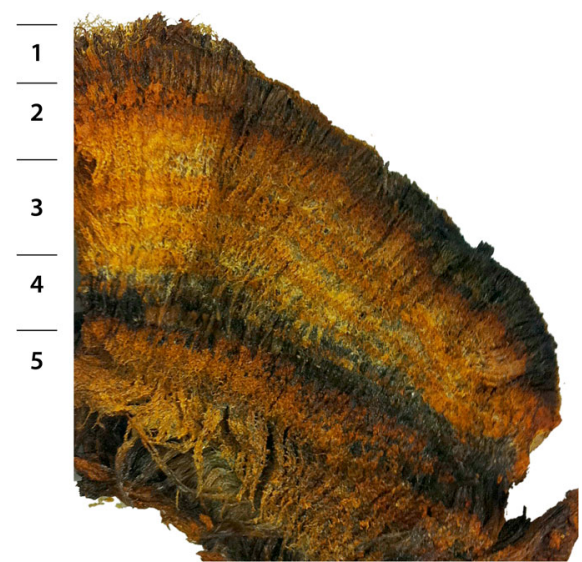

Fig. 3 Cross-section with zones sampled $(1=\mathrm{P} 3,2=\mathrm{P} 4,3=\mathrm{P} 5$, $4=\mathrm{P} 6,5=\mathrm{P} 7)$. Vertical section is about $10 \mathrm{~cm}$ remaining salts in hydrochloric acid (Jackson 1956), elements were estimated using a Perkin Elmer Optima 3000 XL ICP-OES (Perkin Elmer, MA, USA).

- Method 2-samples $(n=1)$ were dried, ignited to destroy organic matter and fused with lithium tetraborate into a bead (Van Reeuwijk 2002). Element concentrations were estimated by X-ray fluorescence spectroscopy (XRF).

- Method 3-total carbon (C), total nitrogen $(\mathrm{N})$ and total sulphur (S) were estimated $(n=2)$ with an Elementar VarioEL elemental analyser (Elementar, Hanau, Germany) according to Van Reeuwijk (2002).

- Method 4-Fe(II) was established by destruction $(n=2)$ in a cold $\mathrm{HF} / \mathrm{H}_{2} \mathrm{SO}_{4}$ solution and determined by colorimetric detection after complexation with 1.10-phenantroline (Van Reeuwijk 2002).

- Method 5-acid ammonium oxalate (AAO) extractable elements at $\mathrm{pH} 3.0$ in the dark were determined $(n=2)$ as described by Van Reeuwijk (2002). In this extraction, all the amorphous material and schwertmannite plus ferrihydrite are dissolved.

Loss on ignition (LOI) was determined $(n=2)$ at $950{ }^{\circ} \mathrm{C}$. X-ray diffraction (XRD) analysis was performed on oriented samples, prepared using a filtermembrane technique. Samples were scanned with a Xray diffractometer with $\mathrm{Cu} \mathrm{K} \alpha$ radiation. The acidified water sample was analysed in duplicate by inductively coupled plasma (ICP) (Perkin Elmer 3000XL OES, MA, USA) and the untreated water sample (single) by Continuous Flow Analyzer SAN++ (Skalar, Breda, the Netherlands). pH was estimated with a WTW pH meter (Weilheim, Germany) and $\mathrm{EC}_{25}$ with a WTW 
conductometer (Weilheim, Germany) with temperature compensation. The VISUAL MINTEQ version 3.0 program (KTH, Stockholm, Sweden) was used to calculate dissolved metal, sulphate and arsenate speciation and to estimate saturation indices for relevant minerals (e.g. Erten-Unal et al. 1998).

\section{Results}

\subsection{Biotic Composition of the Terrace Rims}

During the field sampling in November 2010, we found the terrace rims to be composed of a single moss species. Microscopic study of the terrace rim sample confirmed our field observation on the plant species composition, only one moss species being present. Since the moss had no fructifications, we repeatedly revisited the site over the next 2 years to see whether fructifications were present and eventually collect these for identification, but they were not encountered.

The identification of the moss as Anomobryum prostratum (Müll. Hal.) Besch, by William R. Buck (New York Botanical Garden), was based on morphological characteristics. Confirmation of this identification by molecular genetic data was impossible, since these do not yet exist for the genus (see, e.g. GenBank: www.ncbi. nlm.nih.gov/genbank/). The sample identified has been deposited in the herbarium of the New York Botanical Garden. The morphological characteristics of the species (see Figs. 2, 3 and 4) and its distribution are described in considerable detail in the Tropicos archive (Tropicos.org) and in other publications on this species (e.g. Hegewald and Hegewald et al. 1977 and 1985).

A. prostratum is a rare South American species from the Bryaceae family that occurs from Mexico to Argentina, usually at higher altitudes, i.e. between 1800 and 4285 masl (GBIF 2013). This species belongs to a worldwide Anomobryum genus of 50-60 species, which are most common in montane regions in the southern hemisphere, especially in the Neotropics, and generally occur on damp soil and rock (Spence and Ramsey 2002). In the Tropicos archive (Tropicos.org), the habitat of $A$. prostratum is described as 'on wet soil, bare road-cut soil, moist walls, and boulders in forest or along streams', but the species also occurs in hot springs (GBIF 2013). Hegewald and Hegewald (1977, 1985) found A. prostratum in 1973 in the nearby Catac area. One of the occurrences was at 4100 masl, and Hegewald and Hegewald (Tropicos.org) reported even higher altitudes for Peru.

Under the microscope, bacterial and algal colonies in the form of films, slimes or other types of colonies were not observed, neither on the surface of the living moss (which is hydrophobic) nor inside the moss-built terrace rim sampled (see also Section 4.2). Neither were microbial structures (bacterial or algal colonies) visible at the magnifications used (up to $\times 500$ ) in the suspended sediment present in the stream water and in the interstitial sediment of the moss rim sampled (see Fig. 5).

\subsection{Terrace Rim Structure and Chemical Composition}

A cross-section of a rim is shown in Fig. 3 evidencing the internal structure. Only the outer part (less than $1 \mathrm{~cm}$ ) consisted of green plant parts, and these were hydrophobic, as can be seen in Fig. 2. The remainder consisted of a dense fibrous mass of (macroscopically) moderately encrusted dead moss fibres (necromass) and, in between the fibres, loose creamy to brownish sediment. Remarkable was the occurrence of alternating lighter and more brownish, darker coloured bands, resulting from slight differences in the colour of the moss encrustations. Thicknesses of individual successions (lighter and darker layers) were of the same order as the thickness of the living moss layer, suggesting a seasonal rhythm (dry-wet season) in the built-up of the moss rim.

Figure 4 illustrates the main characteristics of the moss at microscopic scale. The green plant material is largely covered by a thin translucent coating of creamy to brownish, optically isotic material. Going from the green youngest parts downward, the coating changes from this translucent coating to a relatively dense coating composed of very fine-grained creamy material cementing fine crystalline mineral fragments, with dead plant material inside.

Chemical analyses of green, living moss were evidently hampered by the coatings, which appeared to be hard to remove. A moss sample washed with water had a C content of approx. $24 \%$, acid-washed moss $33 \%$ and samples that were thoroughly pretreated (ultrasonic treatment and washing with demi-water) had slightly over $40 \%$ and a weight loss on ignition of near $95 \%$. Element concentrations of these various moss samples are given in Table 2. Microscopic study revealed that in the thoroughly pretreated samples, the living moss still had some thin translucent coatings, while dense creamy coatings were absent, whereas in the other samples such 


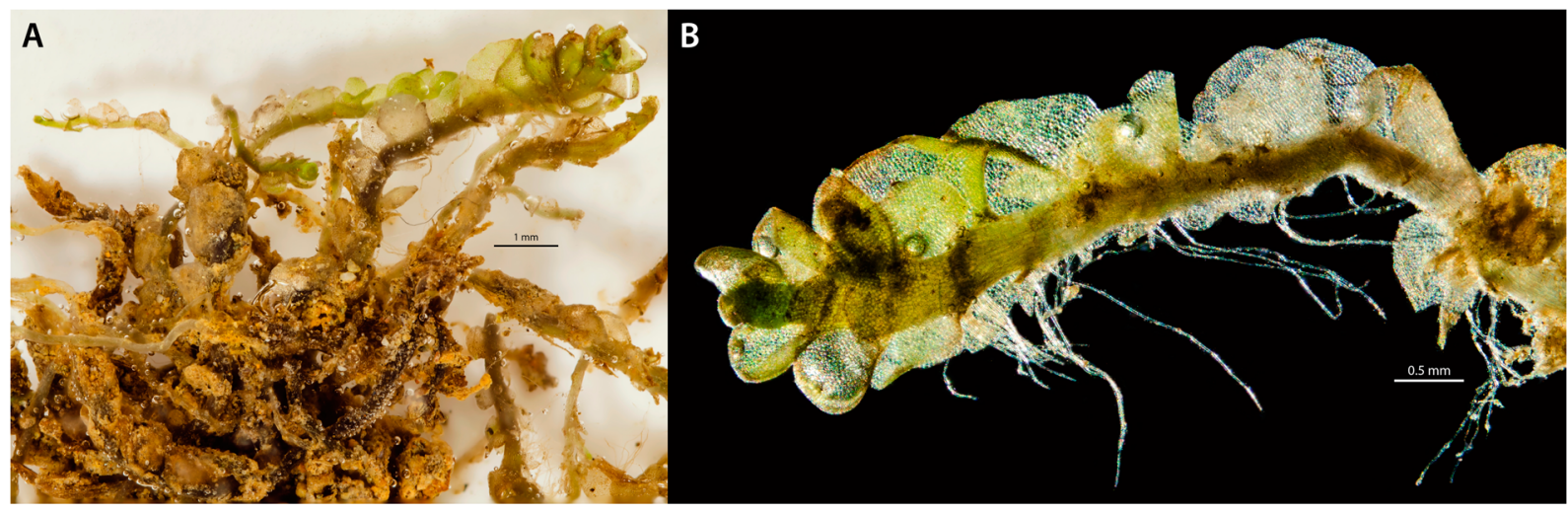

Fig. 4 Microphotographs of the moss showing the development of the coatings: green living moss, thin translucent coatings on still living tissue (b, right side) and thick coatings on dead plant material (a)

relatively dense coatings were common, explaining the observed differences in composition.

\subsection{Water Chemistry}

The data on the water sample taken in 2010 demonstrate the highly acidic nature of the stream water (Table 1). Dissolved $\mathrm{Fe}$ is present in large amounts, as are $\mathrm{Ca}$ and $\mathrm{SO}_{4}$. As to the occurrence of heavy metals and related elements, notable is the concentration of As (104 $\mu \mathrm{mol} \mathrm{L}{ }^{-1}$ ) and the low concentrations of heavy metals with the exception of $\mathrm{Zn}$. Differences in composition relative to the results of Loayza et al. (2010) are evident and primarily concern element concentrations, whereas $\mathrm{pH}$ and $\mathrm{EC}_{25}$ values are similar (indicated with a superscript ' $a$ ' in Table 1). Given the latter, the considerably lower concentration of most elements in the samples of Loayza et al. (2010) is surprising. Unfortunately, their results cannot be checked for their reliability on the basis of the electroneutrality principle, since $\mathrm{S}$ was not estimated. It should be emphasized that

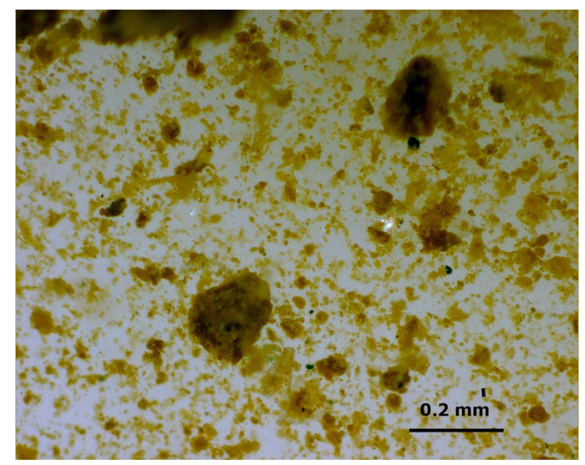

Fig. 5 Microphotograph of suspended material from the stream in the turbulent stream, Loayza et al. (2010) found mean oxygen concentrations of $5 \mathrm{mg} / \mathrm{L}$, which is close to oxygen saturation for this temperature and altitude (Jacobsen 2011) and testifies to the aerobic conditions in the stream.

\subsection{Sediment Composition}

Elemental analyses are presented in Table 3. Fe contents are expressed as $\mathrm{Fe}_{2} \mathrm{O}_{3}, \mathrm{~S}$ as $\mathrm{SO}_{3}$ and $\mathrm{As}$ as $\mathrm{As}_{2} \mathrm{O}_{5}$. $\mathrm{Si}$ contents could not be established by method 1 and therefore were calculated $(\mathrm{Si}=\mathrm{a} * \mathrm{~b})$ from (a) measured $\mathrm{Si} / \mathrm{Al}$ ratios found by XRF (method 2, only performed on samples P1 and P2) and (b) values found for Al by the ICP analyses (method 1). Fe(II) contents of the samples P1-7 (method 4) were in the order of 3-5\% of the total Fe, expressed as oxides, evidencing that virtually all $\mathrm{Fe}$ occurs as $\mathrm{Fe}(\mathrm{III})$. In principle, $\mathrm{S}$ might be present as sulphide, taking into account the nature of the sediment (derived from tailings of sulphidic ores). However, given the prominent aerated nature of the stream water (see, e.g. dissolved oxygen contents in Table 1) and the low concentrations of heavy metals, it is unlikely that more than traces of sulphides are present. Moreover, in the X-ray analyses (samples P1 and P2), no indication was found for the presence of Fe sulphide or other heavy metal sulphides in detectable concentrations. Arsenic is present as $\mathrm{As}_{2} \mathrm{O}_{5}$, since this As occurs as dissolved species in an oxidative environment (Tables 4, 5 and 6).

In Table 3, results from the ammonium oxalate extraction (method 5) of P1 and P2 are presented, providing a measure for the amount and composition of amorphous material. The results show that Fe(III), As and $\mathrm{S}$ 
Table 2 Chemical composition of the moss after different pretreatments (chemical elements in $\mathrm{mmol} \mathrm{kg}^{-1}$ and oxides in weight \%, both on absolute dry base)

\begin{tabular}{|c|c|c|c|c|c|c|c|c|}
\hline & \multicolumn{2}{|l|}{ Moss water } & \multicolumn{2}{|l|}{ Moss acid } & \multicolumn{2}{|c|}{ Moss 1 ultrasonic } & \multicolumn{2}{|c|}{ Moss 2 ultrasonic } \\
\hline & $\mathrm{mmol} \mathrm{kg}{ }^{-1}$ & $\%$ & $\mathrm{mmol} \mathrm{kg}{ }^{-1}$ & $\%$ & $\mathrm{mmol} \mathrm{kg}{ }^{-1}$ & $\%$ & $\mathrm{mmol} \mathrm{kg}{ }^{-1}$ & $\%$ \\
\hline $\mathrm{K} / \mathrm{K}_{2} \mathrm{O}$ & 582 & 2.74 & 566 & 2.67 & 389 & 1.83 & 244 & 1.15 \\
\hline $\mathrm{Na} / \mathrm{Na}_{2} \mathrm{O}$ & 230 & 0.71 & 208 & 0.64 & 92.4 & 0.29 & 52.3 & 0.16 \\
\hline $\mathrm{Ca} / \mathrm{CaO}$ & 98.2 & 0.55 & 62.6 & 0.35 & 136 & 0.76 & 83.7 & 0.47 \\
\hline $\mathrm{Mg} / \mathrm{MgO}$ & 170 & 0.69 & 144 & 0.58 & 73.6 & 0.30 & 61.5 & 0.25 \\
\hline $\mathrm{Al} / \mathrm{Al}_{2} \mathrm{O}_{3}$ & 950 & 4.84 & 642 & 3.27 & 183 & 0.93 & 128 & 0.65 \\
\hline $\mathrm{Fe} / \mathrm{Fe}_{2} \mathrm{O}_{3}$ & 605 & 4.83 & 459 & 3.67 & 153 & 1.22 & 138 & 1.10 \\
\hline $\mathrm{Mn} / \mathrm{MnO}$ & 7.46 & $<<0.1$ & 4.18 & $<<0.1$ & 1.1 & $<<0.1$ & 0.58 & $<<0.1$ \\
\hline $\mathrm{Zn} / \mathrm{ZnO}$ & 16.5 & $<<0.1$ & 11 & $<<0.1$ & 8.9 & $<<0.1$ & 6.67 & $<<0.1$ \\
\hline $\mathrm{Pb} / \mathrm{PbO}$ & 24 & $<<0.1$ & 14 & $<<0.1$ & 3.8 & $<<0.1$ & 2.47 & $<<0.1$ \\
\hline $\mathrm{As} / \mathrm{As}_{2} \mathrm{O}_{5}$ & 88.3 & 1.01 & 81.4 & 0.94 & 10.4 & 0.12 & 7.39 & 0.08 \\
\hline $\mathrm{P} / \mathrm{P}_{2} \mathrm{O}_{5}$ & 40.4 & 0.38 & 45 & 0.43 & 46.1 & 0.44 & 27.9 & 0.26 \\
\hline $\mathrm{S} / \mathrm{SO}_{3}{ }^{\mathrm{a}}$ & 430 & 2.20 & 307 & 1.57 & bdl & bdl & bdl & bdl \\
\hline LOI & & 60.9 & & 70.4 & & 94.0 & & 95.6 \\
\hline $\mathrm{C}^{\mathrm{a}}$ & & 28.7 & & 33.0 & & 40.7 & & 41.2 \\
\hline
\end{tabular}

$b d l$ below detection limit

${ }^{a}$ Based on CNS analyser

are largely present as amorphous material and together comprise about $40-50 \%$ of the sample. Comparing the analyses for $\mathrm{P} 1$ and $\mathrm{P} 2$, it is clear that the latter contains a slightly smaller amorphous component. The X-ray diffraction analyses showed that among the crystalline minerals, quartz, mica and feldspar dominate. This is in line with the total chemical analyses, which combined with the results from the ammonium oxalate extractions evidenced that $\mathrm{SiO}_{2}$ (about $25 \%$ ) and elements such as $\mathrm{Al}_{2} \mathrm{O}_{3}(8-10 \%), \mathrm{K}_{2} \mathrm{O}(1.5 \%), \mathrm{Na}_{2} \mathrm{O}(0.5 \%), \mathrm{MgO}$ $(0.85 \%)$ and $\mathrm{CaO}(0.70 \%)$ are present in the form of crystalline minerals. Reflections from secondary oxyhydroxy sulphate minerals (jarosite, schwertmannite or tooeleite) or iron(hyr)oxides (lepidocrocite, ferrihydrite, goethite) were not observed.

Under the microscope, the suspended material and basal sediment layer (P1 and P2) and the interstitial sediment (P3-P7) appear as mostly silt to fine sandsize light yellow to yellowish brown cryptocrystalline material, which is slightly translucent in fine particles and semi-translucent to isotic in larger aggregates. Particles are equidimensional to irregular and have no microscopically observable internal structure (see Fig. 5) nor features indicative of the presence of cellular or multicellular organisms and their organic structural remains (e.g. cell walls). Angular detrital mineral fragments (mostly quartz, feldspar and mica), also of fine silt to fine sand size, are common as single grains and as embedded fragments in the cryptocrystalline aggregates.

\section{Discussion}

\subsection{Sediment}

The chemical data on the composition of the amorphous material evidence that this material consists of ferric iron oxyhydroxy sulphate minerals that are amorphous to poorly crystalline. Jarosite can be excluded since it would show up in the X-ray analysis and be evident from the relatively high $\mathrm{K}$ content of the amorphous material, which is not the case. In this distinctly acidic environment and with such composition, the occurrence of ferrihydrite in more than very subordinate amounts is improbable (see, e.g. España 2007) and it would show up in a distinctly brown colour of the sediment, which is not the case at all. Therefore, the only relevant minerals are schwertmannite (Bigham et al. 1990, 1996; Yu et al. 1999; Schwertmann and Cornell 2000), the related 
Table 3 Chemical composition of the sediment samples (weight \% or ppm on absolute dry base) based on methods 1 (total) and 5 (AAO: amorph), unless indicated otherwise

\begin{tabular}{|c|c|c|c|c|c|c|c|c|c|}
\hline & \multicolumn{2}{|l|}{$\mathrm{P} 1$} & \multicolumn{2}{|l|}{$\mathrm{P} 2$} & \multirow{2}{*}{$\begin{array}{l}\text { P3 } \\
\text { Total }\end{array}$} & \multirow{2}{*}{$\begin{array}{l}\text { P4 } \\
\text { Total }\end{array}$} & \multirow{2}{*}{$\begin{array}{l}\text { P5 } \\
\text { Total }\end{array}$} & \multirow{2}{*}{$\begin{array}{l}\text { P6 } \\
\text { Total }\end{array}$} & \multirow{2}{*}{$\begin{array}{l}\text { P7 } \\
\text { Total }\end{array}$} \\
\hline & Total & Amorph & Total & Amorph & & & & & \\
\hline \multicolumn{10}{|l|}{ Weight \% } \\
\hline $\mathrm{SiO}_{2}^{\mathrm{a}}$ & 22.6 & 0.07 & 26.5 & 0.10 & nd & nd & nd & nd & nd \\
\hline $\mathrm{K}_{2} \mathrm{O}$ & 1.63 & 0.04 & 1.69 & 0.01 & 0.51 & 1.69 & 1.61 & 1.04 & 1.50 \\
\hline $\mathrm{Na}_{2} \mathrm{O}$ & 0.52 & $<0.01$ & 0.51 & $<0.01$ & 0.10 & 0.49 & 0.53 & 0.21 & 0.49 \\
\hline $\mathrm{CaO}$ & 0.71 & 0.01 & 0.68 & 0.01 & 0.38 & 0.82 & 0.96 & 0.48 & 0.75 \\
\hline $\mathrm{MgO}$ & 0.84 & 0.02 & 0.87 & 0.01 & 0.29 & 0.82 & 0.81 & 0.47 & 0.68 \\
\hline $\mathrm{Al}_{2} \mathrm{O}_{3}$ & 8.58 & 0.11 & 9.29 & 0.25 & 2.62 & 8.26 & 8.51 & 5.39 & 7.98 \\
\hline $\mathrm{Fe}_{2} \mathrm{O}_{3}$ & 33.2 & 23.4 & 29.2 & 21.1 & 45.6 & 30.0 & 27.6 & 32.8 & 31.1 \\
\hline $\mathrm{TiO}_{2}$ & 0.40 & $<0.01$ & 0.41 & $<0.01$ & nd & nd & nd & nd & nd \\
\hline $\mathrm{P}_{2} \mathrm{O}_{5}$ & 0.35 & 0.22 & 0.39 & 0.28 & $<0.01$ & 0.35 & 0.36 & bdl & 0.47 \\
\hline $\mathrm{SO}_{3}{ }^{\mathrm{b}}$ & 6.93 & $3.44^{\mathrm{c}}$ & 5.55 & $2.68^{\mathrm{c}}$ & 6.61 & 6.53 & 7.44 & 8.01 & 6.23 \\
\hline FeO by method 4 & 0.91 & & 1.12 & & 2.28 & 1.17 & 1.09 & 1.27 & 0.83 \\
\hline \multicolumn{10}{|l|}{ Content in ppm } \\
\hline $\mathrm{Mn}$ & 438 & 22 & 657 & 107 & 785 & 653 & 645 & 664 & 575 \\
\hline $\mathrm{Zn}$ & 1910 & 97 & 2120 & 346 & 2980 & 1630 & 1280 & 1350 & 1330 \\
\hline $\mathrm{Pb}$ & 4450 & nd & 5400 & nd & 1340 & 3440 & 5350 & 3530 & 5500 \\
\hline $\mathrm{Cu}$ & 150 & 20 & 159 & 31 & 79 & 204 & 321 & 651 & 369 \\
\hline $\mathrm{Sr}$ & 81 & nd & 91 & nd & 34 & 97 & 106 & 47 & 91 \\
\hline As & 21,400 & 15,700 & 24,800 & 17,900 & 12,300 & 17,800 & 23,600 & 20,200 & 36,500 \\
\hline \multicolumn{10}{|l|}{ Weight \% } \\
\hline $\mathrm{C}^{\mathrm{b}}$ & 1.99 & & 1.95 & & 12.17 & 2.75 & 1.86 & 11.95 & 3.22 \\
\hline $\mathrm{N}^{\mathrm{b}}$ & 0.20 & & 0.16 & & 0.72 & 0.23 & 0.15 & 0.49 & 0.18 \\
\hline $\mathrm{S}^{\mathrm{b}}$ & 2.77 & & 2.22 & & 3.13 & 2.51 & 3.10 & 2.83 & 2.32 \\
\hline LOI & 15.50 & & 12.52 & & 28.63 & 10.24 & 7.50 & 29.35 & 10.42 \\
\hline
\end{tabular}

Values for elements are expressed in at most three significant digits

$n d$ not determined

${ }^{\text {a }}$ Calculated from X-ray fluorescence

${ }^{\mathrm{b}} \mathrm{CNS}$ analyser

${ }^{\mathrm{c}}$ By ICP

tooeleite (Morin et al. 2003; Nishimura and Robins 2008) or a mixture of both. It should be emphasized that poorly crystalline schwertmannite and tooeleite cannot be readily identified through X-ray diffractometry, for which reason we describe this compound as schwertmannite-type material. Most of the total As ( $73 \%$ as $\mathrm{As}_{2} \mathrm{O}_{5}$ ) also dissolved in the $\mathrm{NH}_{4} \mathrm{Ox} / \mathrm{Ox}$ extract. Whether this As is bound in the structure (both tooeleite and schwertmannite) or sorbed as arsenate (schwertmannite) is not clear, but sorption is well known and considered to be most likely (Murad et al. 1994;
Waychunas et al. 1995; Carlson et al. 2002; Fukushi et al. 2003).

The results from method 5 show that amorphous $\mathrm{Al}$ and $\mathrm{Si}$ is hardly present, virtually all $\mathrm{Si}$ and $\mathrm{Al}$ being present in the form of crystalline mineral fragments. The same holds for the base metals $(\mathrm{K}, \mathrm{Na}, \mathrm{Mg}$ and $\mathrm{Ca}$ ). $\mathrm{P}$ is clearly present in very minor amounts. Thus, about half of the sediment (P1 and P2) consists of schwertmannitetype material with an appreciable arsenate component. The other half consists of a fine-grained mixture of primary quartz, mica and feldspar with minor amounts 
Table 4 Log IAP, sat. index and stoichiometry of minerals

\begin{tabular}{|c|c|c|c|c|c|c|c|c|c|c|c|c|}
\hline \multirow{2}{*}{$\begin{array}{l}\text { Mineral } \\
\mathrm{Al}(\mathrm{OH})_{3}(\text { soil })\end{array}$} & \multirow{2}{*}{$\frac{\log \text { IAP }}{2.9}$} & \multirow{2}{*}{$\frac{\text { Sat. index }}{-6.262}$} & \multicolumn{10}{|c|}{ Stoichiometry } \\
\hline & & & 1 & $\mathrm{Al}^{+3}$ & 3 & $\mathrm{H}_{2} \mathrm{O}$ & -3 & $\mathrm{H}^{+1}$ & & & & \\
\hline $\mathrm{Al}_{2} \mathrm{O}_{3}(\mathrm{~s})$ & 5.8 & -16.001 & 2 & $\mathrm{Al}^{+3}$ & 3 & $\mathrm{H}_{2} \mathrm{O}$ & -6 & $\mathrm{H}^{+1}$ & & & & \\
\hline $\mathrm{Al}_{4}(\mathrm{OH})_{10} \mathrm{SO}_{4}(\mathrm{~s})$ & 2.496 & -20.204 & -10 & $\mathrm{H}^{+1}$ & 4 & $\mathrm{Al}^{+3}$ & 1 & $\mathrm{SO}_{4}^{-2}$ & 10 & $\mathrm{H}_{2} \mathrm{O}$ & & \\
\hline $\mathrm{AlAsO}_{4}:_{2} \mathrm{H}_{2} \mathrm{O}(\mathrm{s})$ & -23.45 & -7.65 & 1 & $\mathrm{Al}^{+3}$ & 1 & $\mathrm{AsO}_{4}^{-3}$ & 2 & $\mathrm{H}_{2} \mathrm{O}$ & & & & \\
\hline $\mathrm{AlOHSO}_{4}(\mathrm{~s})$ & -6.204 & -2.974 & -1 & $\mathrm{H}^{+1}$ & 1 & $\mathrm{Al}^{+3}$ & 1 & $\mathrm{SO}_{4}^{-2}$ & 1 & $\mathrm{H}_{2} \mathrm{O}$ & & \\
\hline Alunite & -10.228 & -10.573 & 1 & $\mathrm{~K}^{+1}$ & 3 & $\mathrm{Al}^{+3}$ & 2 & $\mathrm{SO}_{4}^{-2}$ & -6 & $\mathrm{H}^{+1}$ & 6 & $\mathrm{H}_{2} \mathrm{O}$ \\
\hline Anglesite & -9.649 & -1.759 & 1 & $\mathrm{~Pb}^{+2}$ & 1 & $\mathrm{SO}_{4}^{-2}$ & & & & & & \\
\hline Anhydrite & -5.293 & -0.993 & 1 & $\mathrm{Ca}^{+2}$ & 1 & $\mathrm{SO}_{4}^{-2}$ & & & & & & \\
\hline Antlerite & -13.6 & -22.388 & 3 & $\mathrm{Cu}^{+2}$ & 4 & $\mathrm{H}_{2} \mathrm{O}$ & -4 & $\mathrm{H}^{+1}$ & 1 & $\mathrm{SO}_{4}^{-2}$ & & \\
\hline Aragonite & -29.105 & -20.843 & 1 & $\mathrm{Ca}^{+2}$ & 1 & $\mathrm{CO}_{3}^{-2}$ & & & & & & \\
\hline Artinite & -26.532 & -37.131 & -2 & $\mathrm{H}^{+1}$ & 2 & $\mathrm{Mg}^{+2}$ & 1 & $\mathrm{CO}_{3}^{-2}$ & 5 & $\mathrm{H}_{2} \mathrm{O}$ & & \\
\hline $\mathrm{As}_{2} \mathrm{O}_{5}(\mathrm{~s})$ & -52.7 & -17.983 & 2 & $\mathrm{AsO}_{4}^{-3}$ & 6 & $\mathrm{H}^{+1}$ & -3 & $\mathrm{H}_{2} \mathrm{O}$ & & & & \\
\hline Atacamite & -9.97 & -18.137 & 2 & $\mathrm{Cu}^{+2}$ & 3 & $\mathrm{H}_{2} \mathrm{O}$ & -3 & $\mathrm{H}^{+1}$ & 1 & $\mathrm{Cl}^{-1}$ & & \\
\hline Azurite & -70.328 & -53.265 & 3 & $\mathrm{Cu}^{+2}$ & 2 & $\mathrm{H}_{2} \mathrm{O}$ & -2 & $\mathrm{H}^{+1}$ & 2 & $\mathrm{CO}_{3}^{-2}$ & & \\
\hline Bianchite & -5.898 & -4.138 & 1 & $\mathrm{Zn}^{+2}$ & 1 & $\mathrm{SO}_{4}^{-2}$ & 6 & $\mathrm{H}_{2} \mathrm{O}$ & & & & \\
\hline Boehmite & 2.9 & -6.656 & -3 & $\mathrm{H}^{+1}$ & 1 & $\mathrm{Al}^{+3}$ & 2 & $\mathrm{H}_{2} \mathrm{O}$ & & & & \\
\hline Brochantite & -15.098 & -32.006 & 4 & $\mathrm{Cu}^{+2}$ & 6 & $\mathrm{H}_{2} \mathrm{O}$ & -6 & $\mathrm{H}^{+1}$ & 1 & $\mathrm{SO}_{4}^{-2}$ & & \\
\hline Brucite & 3.192 & -14.855 & 1 & $\mathrm{Mg}^{+2}$ & 2 & $\mathrm{H}_{2} \mathrm{O}$ & -2 & $\mathrm{H}^{+1}$ & & & & \\
\hline $\mathrm{Ca}_{3}\left(\mathrm{AsO}_{4}\right)_{2}: \mathrm{H}_{2} \mathrm{O}(\mathrm{s})$ & -41.267 & -22.367 & 3 & $\mathrm{Ca}^{+2}$ & 2 & $\mathrm{AsO}_{4}^{-3}$ & 4 & $\mathrm{H}_{2} \mathrm{O}$ & & & & \\
\hline $\mathrm{Ca}_{3}\left(\mathrm{PO}_{4}\right)_{2}\left(\mathrm{am}_{1}\right)$ & -49.986 & -25.267 & 3 & $\mathrm{Ca}^{+2}$ & 2 & $\mathrm{PO}_{4}^{-3}$ & -3 & $\mathrm{H}^{+1}$ & & & & \\
\hline $\mathrm{Ca}_{3}\left(\mathrm{PO}_{4}\right)_{2}\left(\mathrm{am}_{2}\right)$ & -49.986 & -22.459 & 3 & $\mathrm{Ca}^{+2}$ & 2 & $\mathrm{PO}_{4}^{-3}$ & -3 & $\mathrm{H}^{+1}$ & & & & \\
\hline $\mathrm{Ca}_{3}\left(\mathrm{PO}_{4}\right)_{2}$ (beta) & -49.986 & -20.617 & 3 & $\mathrm{Ca}^{+2}$ & 2 & $\mathrm{PO}_{4}^{-3}$ & & & & & & \\
\hline $\mathrm{Ca}_{4} \mathrm{H}\left(\mathrm{PO}_{4}\right)_{3}:{ }_{3} \mathrm{H}_{2} \mathrm{O}(\mathrm{s})$ & -76.884 & -29.806 & 4 & $\mathrm{Ca}^{+2}$ & 1 & $\mathrm{H}^{+1}$ & 3 & $\mathrm{PO}_{4}^{-3}$ & 3 & $\mathrm{H}_{2} \mathrm{O}$ & & \\
\hline $\mathrm{CaCO}_{3} \mathrm{xH}_{2} \mathrm{O}(\mathrm{s})$ & -29.105 & -22.03 & 1 & $\mathrm{Ca}^{+2}$ & 1 & $\mathrm{CO}_{3}^{-2}$ & 1 & $\mathrm{H}_{2} \mathrm{O}$ & & & & \\
\hline $\mathrm{CaHPO}_{4}(\mathrm{~s})$ & -26.898 & -7.366 & 1 & $\mathrm{Ca}^{+2}$ & 1 & $\mathrm{H}^{+1}$ & 1 & $\mathrm{PO}_{4}^{-3}$ & & & & \\
\hline $\mathrm{CaHPO}_{4}:_{2} \mathrm{H}_{2} \mathrm{O}(\mathrm{s})$ & -26.898 & -7.712 & 1 & $\mathrm{Ca}^{+2}$ & 1 & $\mathrm{H}^{+1}$ & 1 & $\mathrm{PO}_{4}^{-3}$ & 2 & $\mathrm{H}_{2} \mathrm{O}$ & & \\
\hline Calcite & -29.105 & -20.689 & 1 & $\mathrm{Ca}^{+2}$ & 1 & $\mathrm{CO}_{3}^{-2}$ & & & & & & \\
\hline $\mathrm{Cd}(\mathrm{OH})_{2}(\mathrm{~s})$ & -0.214 & -14.644 & 1 & $\mathrm{Cd}^{+2}$ & 2 & $\mathrm{H}_{2} \mathrm{O}$ & -2 & $\mathrm{H}^{+1}$ & & & & \\
\hline $\mathrm{Cd}_{3}(\mathrm{OH})_{4} \mathrm{SO}_{4}(\mathrm{~s})$ & -9.745 & -32.305 & -4 & $\mathrm{H}^{+1}$ & 3 & $\mathrm{Cd}^{+2}$ & 4 & $\mathrm{H}_{2} \mathrm{O}$ & 1 & $\mathrm{SO}_{4}{ }^{-2}$ & & \\
\hline $\mathrm{Cd}_{3}\left(\mathrm{PO}_{4}\right)_{2}(\mathrm{~s})$ & -62.059 & -29.459 & 3 & $\mathrm{Cd}^{+2}$ & 2 & $\mathrm{PO}_{4}^{-3}$ & & & & & & \\
\hline $\mathrm{Cd}_{3} \mathrm{OH}_{2}\left(\mathrm{SO}_{4}\right)_{2}(\mathrm{~s})$ & -18.849 & -25.559 & -2 & $\mathrm{H}^{+1}$ & 3 & $\mathrm{Cd}^{+2}$ & 2 & $\mathrm{H}_{2} \mathrm{O}$ & 2 & $\mathrm{SO}_{4}^{-2}$ & & \\
\hline $\mathrm{Cd}_{4}(\mathrm{OH})_{6} \mathrm{SO}_{4}(\mathrm{~s})$ & -9.959 & -38.359 & -6 & $\mathrm{H}^{+1}$ & 4 & $\mathrm{Cd}^{+2}$ & 6 & $\mathrm{H}_{2} \mathrm{O}$ & 1 & $\mathrm{SO}_{4}^{-2}$ & & \\
\hline $\mathrm{CdCl}_{2}(\mathrm{~s})$ & -14.159 & -13.654 & 1 & $\mathrm{Cd}^{+2}$ & 2 & $\mathrm{Cl}^{-1}$ & & & & & & \\
\hline $\mathrm{CdCl}_{2: 1} \mathrm{H}_{2} \mathrm{O}(\mathrm{s})$ & -14.159 & -12.528 & 1 & $\mathrm{Cd}^{+2}$ & 2 & $\mathrm{Cl}^{-1}$ & 1 & $\mathrm{H}_{2} \mathrm{O}$ & & & & \\
\hline $\mathrm{CdCl}_{2} \cdot 2 \cdot 5 \mathrm{H}_{2} \mathrm{O}(\mathrm{s})$ & -14.159 & -12.185 & 1 & $\mathrm{Cd}^{+2}$ & 2 & $\mathrm{Cl}^{-1}$ & 2.5 & $\mathrm{H}_{2} \mathrm{O}$ & & & & \\
\hline $\mathrm{CdOHCl}(\mathrm{s})$ & -7.186 & -10.98 & -1 & $\mathrm{H}^{+1}$ & 1 & $\mathrm{Cd}^{+2}$ & 1 & $\mathrm{H}_{2} \mathrm{O}$ & 1 & $\mathrm{Cl}^{-1}$ & & \\
\hline $\mathrm{CdSO}_{4}(\mathrm{~s})$ & -9.318 & -9.577 & 1 & $\mathrm{Cd}^{+2}$ & 1 & $\mathrm{SO}_{4}^{-2}$ & & & & & & \\
\hline $\mathrm{CdSO}_{4}:_{1} \mathrm{H}_{2} \mathrm{O}(\mathrm{s})$ & -9.318 & -7.854 & 1 & $\mathrm{Cd}^{+2}$ & 1 & $\mathrm{SO}_{4}^{-2}$ & 1 & $\mathrm{H}_{2} \mathrm{O}$ & & & & \\
\hline $\mathrm{CdSO}_{4} \cdot{ }_{2} \cdot{ }_{67} \mathrm{H}_{2} \mathrm{O}(\mathrm{s})$ & -9.318 & -7.594 & 1 & $\mathrm{Cd}^{+2}$ & 1 & $\mathrm{SO}_{4}^{-2}$ & 2.67 & $\mathrm{H}_{2} \mathrm{O}$ & & & & \\
\hline Celestite & -7.842 & -1.205 & 1 & $\mathrm{Sr}^{+2}$ & 1 & $\mathrm{SO}_{4}^{-2}$ & & & & & & \\
\hline Cerussite & -33.461 & -20.055 & 1 & $\mathrm{~Pb}^{+2}$ & 1 & $\mathrm{CO}_{3}^{-2}$ & & & & & & \\
\hline Chalcanthite & -10.603 & -7.913 & 1 & $\mathrm{Cu}^{+2}$ & 1 & $\mathrm{SO}_{4}^{-2}$ & 5 & $\mathrm{H}_{2} \mathrm{O}$ & & & & \\
\hline Chalcedony & -3.465 & 0.249 & 1 & $\mathrm{H}_{4} \mathrm{SiO}_{4}$ & -2 & $\mathrm{H}_{2} \mathrm{O}$ & & & & & & \\
\hline Chloropyromorphite(c) & -101.825 & -17.395 & 5 & $\mathrm{~Pb}^{+2}$ & 3 & $\mathrm{PO}_{4}^{-3}$ & 1 & $\mathrm{Cl}^{-1}$ & & & & \\
\hline
\end{tabular}


Table 4 (continued)

\begin{tabular}{|c|c|c|c|c|c|c|c|c|c|c|c|c|}
\hline \multirow{2}{*}{$\begin{array}{l}\text { Mineral } \\
\text { Chloropyromorphite(soil) }\end{array}$} & \multirow{2}{*}{$\begin{array}{l}\text { Log IAP } \\
-101.825\end{array}$} & \multirow{2}{*}{$\begin{array}{l}\text { Sat. index } \\
-21.425\end{array}$} & \multicolumn{10}{|c|}{ Stoichiometry } \\
\hline & & & 5 & $\mathrm{~Pb}^{+2}$ & 3 & $\mathrm{PO}_{4}^{-3}$ & 1 & $\mathrm{Cl}^{-1}$ & & & & \\
\hline Chrysotile & 2.646 & -31.182 & 3 & $\mathrm{Mg}^{+2}$ & 2 & $\mathrm{H}_{4} \mathrm{SiO}_{4}$ & 1 & $\mathrm{H}_{2} \mathrm{O}$ & -6 & $\mathrm{H}^{+1}$ & & \\
\hline Cotunnite & -14.49 & -9.493 & 1 & $\mathrm{~Pb}^{+2}$ & 2 & $\mathrm{Cl}^{-1}$ & & & & & & \\
\hline Cristobalite & -3.465 & 0.051 & 1 & $\mathrm{H}_{4} \mathrm{SiO}_{4}$ & -2 & $\mathrm{H}_{2} \mathrm{O}$ & & & & & & \\
\hline $\mathrm{Cu}(\mathrm{OH})_{2}(\mathrm{~s})$ & -1.499 & -11.23 & 1 & $\mathrm{Cu}^{+2}$ & 2 & $\mathrm{H}_{2} \mathrm{O}$ & -2 & $\mathrm{H}^{+1}$ & & & & \\
\hline $\mathrm{Cu}_{2}(\mathrm{OH})_{3} \mathrm{NO}_{3}(\mathrm{~s})$ & -12.893 & -22.747 & 2 & $\mathrm{Cu}^{+2}$ & 3 & $\mathrm{H}_{2} \mathrm{O}$ & -3 & $\mathrm{H}^{+1}$ & 1 & $\mathrm{NO}_{3}{ }^{-1}$ & & \\
\hline $\mathrm{Cu}_{3}\left(\mathrm{AsO}_{4}\right)_{2}: \mathrm{H}_{2} \mathrm{O}(\mathrm{s})$ & -57.195 & -22.095 & 3 & $\mathrm{Cu}^{+2}$ & 2 & $\mathrm{AsO}_{4}^{-3}$ & 2 & $\mathrm{H}_{2} \mathrm{O}$ & & & & \\
\hline $\mathrm{Cu}_{3}\left(\mathrm{PO}_{4}\right)_{2}(\mathrm{~s})$ & -65.914 & -29.064 & 3 & $\mathrm{Cu}^{+2}$ & 2 & $\mathrm{PO}_{4}^{-3}$ & & & & & & \\
\hline $\mathrm{Cu}_{3}\left(\mathrm{PO}_{4}\right)_{2}:{ }_{3} \mathrm{H}_{2} \mathrm{O}(\mathrm{s})$ & -65.914 & -30.794 & 3 & $\mathrm{Cu}^{+2}$ & 2 & $\mathrm{PO}_{4}^{-3}$ & 3 & $\mathrm{H}_{2} \mathrm{O}$ & & & & \\
\hline $\mathrm{CuCO}_{3}(\mathrm{~s})$ & -34.415 & -22.915 & 1 & $\mathrm{Cu}^{+2}$ & 1 & $\mathrm{CO}_{3}^{-2}$ & & & & & & \\
\hline $\mathrm{CuOCuSO}_{4}(\mathrm{~s})$ & -12.101 & -23.549 & -2 & $\mathrm{H}^{+1}$ & 2 & $\mathrm{Cu}^{+2}$ & 1 & $\mathrm{H}_{2} \mathrm{O}$ & 1 & $\mathrm{SO}_{4}^{-2}$ & & \\
\hline Cupric ferrite & 9.832 & 2.097 & -8 & $\mathrm{H}^{+1}$ & 1 & $\mathrm{Cu}^{+2}$ & 2 & $\mathrm{Fe}^{+3}$ & 4 & $\mathrm{H}_{2} \mathrm{O}$ & & \\
\hline $\mathrm{CuSO}_{4}(\mathrm{~s})$ & -10.603 & -14.149 & 1 & $\mathrm{Cu}^{+2}$ & 1 & $\mathrm{SO}_{4}^{-2}$ & & & & & & \\
\hline Diaspore & 2.9 & -4.829 & -3 & $\mathrm{H}^{+1}$ & 1 & $\mathrm{Al}^{+3}$ & 2 & $\mathrm{H}_{2} \mathrm{O}$ & & & & \\
\hline Dolomite (disordered) & -58.829 & -42.675 & 1 & $\mathrm{Ca}^{+2}$ & 1 & $\mathrm{Mg}^{+2}$ & 2 & $\mathrm{CO}_{3}^{-2}$ & & & & \\
\hline Dolomite (ordered) & -58.829 & -42.067 & 1 & $\mathrm{Ca}^{+2}$ & 1 & $\mathrm{Mg}^{+2}$ & 2 & $\mathrm{CO}_{3}^{-2}$ & & & & \\
\hline Epsomite & -5.912 & -3.689 & 1 & $\mathrm{Mg}^{+2}$ & 1 & $\mathrm{SO}_{4}^{-2}$ & 7 & $\mathrm{H}_{2} \mathrm{O}$ & & & & \\
\hline Ettringite & 1.353 & -58.693 & 6 & $\mathrm{Ca}^{+2}$ & 2 & $\mathrm{Al}^{+3}$ & 3 & $\mathrm{SO}_{4}^{-2}$ & -12 & $\mathrm{H}^{+1}$ & 38 & $\mathrm{H}_{2} \mathrm{O}$ \\
\hline $\mathrm{Fe}(\mathrm{OH})_{2}(\mathrm{am})$ & 2.891 & -11.361 & 1 & $\mathrm{Fe}^{+2}$ & 2 & $\mathrm{H}_{2} \mathrm{O}$ & -2 & $\mathrm{H}^{+1}$ & & & & \\
\hline $\mathrm{Fe}(\mathrm{OH})_{2}(\mathrm{c})$ & 2.891 & -9.999 & 1 & $\mathrm{Fe}^{+2}$ & -2 & $\mathrm{H}^{+1}$ & 2 & $\mathrm{H}_{2} \mathrm{O}$ & & & & \\
\hline $\mathrm{Fe}(\mathrm{OH})_{2 \cdot 7} \mathrm{Cl}_{\cdot 3}(\mathrm{~s})$ & 3.574 & 6.614 & -2.7 & $\mathrm{H}^{+1}$ & 1 & $\mathrm{Fe}^{+3}$ & 2.7 & $\mathrm{H}_{2} \mathrm{O}$ & 0.3 & $\mathrm{Cl}^{-1}$ & & \\
\hline $\mathrm{Fe}_{2}\left(\mathrm{SO}_{4}\right)_{3}(\mathrm{~s})$ & -15.981 & -14.258 & 2 & $\mathrm{Fe}^{+3}$ & 3 & $\mathrm{SO}_{4}^{-2}$ & & & & & & \\
\hline $\mathrm{Fe}_{3}(\mathrm{OH})_{8}(\mathrm{~s})$ & 14.221 & -6.001 & -8 & $\mathrm{H}^{+1}$ & 2 & $\mathrm{Fe}^{+3}$ & 1 & $\mathrm{Fe}^{+2}$ & 8 & $\mathrm{H}_{2} \mathrm{O}$ & & \\
\hline $\mathrm{FeAsO}_{4}:_{2} \mathrm{H}_{2} \mathrm{O}(\mathrm{s})$ & -20.684 & -0.484 & 1 & $\mathrm{Fe}^{+3}$ & 1 & $\mathrm{AsO}_{4}^{-3}$ & 2 & $\mathrm{H}_{2} \mathrm{O}$ & & & & \\
\hline Ferrihydrite & 5.665 & 1.631 & 1 & $\mathrm{Fe}^{+3}$ & 3 & $\mathrm{H}_{2} \mathrm{O}$ & -3 & $\mathrm{H}^{+1}$ & & & & \\
\hline Ferrihydrite (aged) & 5.665 & 2.141 & 1 & $\mathrm{Fe}^{+3}$ & -3 & $\mathrm{H}^{+1}$ & 3 & $\mathrm{H}_{2} \mathrm{O}$ & & & & \\
\hline Gibbsite (C) & 2.9 & -5.712 & 1 & $\mathrm{Al}^{+3}$ & 3 & $\mathrm{H}_{2} \mathrm{O}$ & -3 & $\mathrm{H}^{+1}$ & & & & \\
\hline Goethite & 5.665 & 4.671 & 1 & $\mathrm{Fe}^{+3}$ & 2 & $\mathrm{H}_{2} \mathrm{O}$ & -3 & $\mathrm{H}^{+1}$ & & & & \\
\hline Goslarite & -5.898 & -3.769 & 1 & $\mathrm{Zn}^{+2}$ & 1 & $\mathrm{SO}_{4}^{-2}$ & 7 & $\mathrm{H}_{2} \mathrm{O}$ & & & & \\
\hline Greenalite & 1.742 & -19.068 & -6 & $\mathrm{H}^{+1}$ & 3 & $\mathrm{Fe}^{+2}$ & 2 & $\mathrm{H}_{4} \mathrm{SiO}_{4}$ & 1 & $\mathrm{H}_{2} \mathrm{O}$ & & \\
\hline Gypsum & -5.293 & -0.675 & 1 & $\mathrm{Ca}^{+2}$ & 1 & $\mathrm{SO}_{4}^{-2}$ & 2 & $\mathrm{H}_{2} \mathrm{O}$ & & & & \\
\hline Halite & -6.992 & -8.511 & 1 & $\mathrm{Na}^{+1}$ & 1 & $\mathrm{Cl}^{-1}$ & & & & & & \\
\hline Halloysite & -1.13 & -12.212 & 2 & $\mathrm{Al}^{+3}$ & 2 & $\mathrm{H}_{4} \mathrm{SiO}_{4}$ & 1 & $\mathrm{H}_{2} \mathrm{O}$ & -6 & $\mathrm{H}^{+1}$ & & \\
\hline Hematite & 11.331 & 11.677 & 2 & $\mathrm{Fe}^{+3}$ & 3 & $\mathrm{H}_{2} \mathrm{O}$ & -6 & $\mathrm{H}^{+1}$ & & & & \\
\hline Hercynite & 8.691 & -16.81 & -8 & $\mathrm{H}^{+1}$ & 1 & $\mathrm{Fe}^{+2}$ & 2 & $\mathrm{Al}^{+3}$ & 4 & $\mathrm{H}_{2} \mathrm{O}$ & & \\
\hline Hinsdalite & -31.658 & -29.158 & -6 & $\mathrm{H}^{+1}$ & 1 & $\mathrm{~Pb}^{+2}$ & 3 & $\mathrm{Al}^{+3}$ & 1 & $\mathrm{PO}_{4}^{-3}$ & 1 & $\mathrm{SO}_{4}^{-2}$ \\
\hline H-Jarosite & -1.212 & 2.323 & 3 & $\mathrm{Fe}^{+3}$ & 2 & $\mathrm{SO}_{4}^{-2}$ & -5 & $\mathrm{H}^{+1}$ & 7 & $\mathrm{H}_{2} \mathrm{O}$ & & \\
\hline Huntite & -118.277 & -89.205 & 3 & $\mathrm{Mg}^{+2}$ & 1 & $\mathrm{Ca}^{+2}$ & 4 & $\mathrm{CO}_{3}^{-2}$ & & & & \\
\hline Hydrocerrusite & -67.467 & -48.707 & 3 & $\mathrm{~Pb}^{+2}$ & 2 & $\mathrm{H}_{2} \mathrm{O}$ & -2 & $\mathrm{H}^{+1}$ & 2 & $\mathrm{CO}_{3}^{-2}$ & & \\
\hline Hydromagnesite & -115.704 & -108.753 & 5 & $\mathrm{Mg}^{+2}$ & 4 & $\mathrm{CO}_{3}^{-2}$ & -2 & $\mathrm{H}^{+1}$ & 6 & $\mathrm{H}_{2} \mathrm{O}$ & & \\
\hline Hydroxyapatite & -73.073 & -28.74 & 5 & $\mathrm{Ca}^{+2}$ & 3 & $\mathrm{PO}_{4}^{-3}$ & 1 & $\mathrm{H}_{2} \mathrm{O}$ & -1 & $\mathrm{H}^{+1}$ & & \\
\hline Hydroxylpyromorphite & -94.852 & -32.062 & 5 & $\mathrm{~Pb}^{+2}$ & 3 & $\mathrm{PO}_{4}^{-3}$ & 1 & $\mathrm{H}_{2} \mathrm{O}$ & -1 & $\mathrm{H}^{+1}$ & & \\
\hline Hydrozincite & -49.801 & -60.466 & 5 & $\mathrm{Zn}^{+2}$ & 2 & $\mathrm{CO}_{3}^{-2}$ & -6 & $\mathrm{H}^{+1}$ & 6 & $\mathrm{H}_{2} \mathrm{O}$ & & \\
\hline Imogolite & 2.335 & -12.273 & 2 & $\mathrm{Al}^{+3}$ & 1 & $\mathrm{H}_{4} \mathrm{SiO}_{4}$ & 3 & $\mathrm{H}_{2} \mathrm{O}$ & -6 & $\mathrm{H}^{+1}$ & & \\
\hline
\end{tabular}


Table 4 (continued)

\begin{tabular}{|c|c|c|c|c|c|c|c|c|c|c|c|c|}
\hline \multirow{2}{*}{$\begin{array}{l}\text { Mineral } \\
\text { K-Alum }\end{array}$} & \multirow{2}{*}{$\begin{array}{c}\text { Log IAP } \\
-16.029\end{array}$} & \multirow{2}{*}{$\begin{array}{c}\text { Sat. index } \\
-10.608\end{array}$} & \multicolumn{10}{|c|}{ Stoichiometry } \\
\hline & & & 1 & $\mathrm{~K}^{+1}$ & 1 & $\mathrm{Al}^{+3}$ & 2 & $\mathrm{SO}_{4}^{-2}$ & 12 & $\mathrm{H}_{2} \mathrm{O}$ & & \\
\hline Kaolinite & -1.13 & -9.794 & 2 & $\mathrm{Al}^{+3}$ & 2 & $\mathrm{H}_{4} \mathrm{SiO}_{4}$ & 1 & $\mathrm{H}_{2} \mathrm{O}$ & -6 & $\mathrm{H}^{+1}$ & & \\
\hline $\mathrm{KCl}(\mathrm{s})$ & -7.693 & -8.593 & 1 & $\mathrm{~K}^{+1}$ & 1 & $\mathrm{Cl}^{-1}$ & & & & & & \\
\hline K-Jarosite & -1.932 & 8.222 & 1 & $\mathrm{~K}^{+1}$ & 3 & $\mathrm{Fe}^{+3}$ & 2 & $\mathrm{SO}_{4}{ }^{-2}$ & -6 & $\mathrm{H}^{+1}$ & 6 & $\mathrm{H}_{2} \mathrm{O}$ \\
\hline Langite & -15.098 & -33.962 & -6 & $\mathrm{H}^{+1}$ & 4 & $\mathrm{Cu}^{+2}$ & 7 & $\mathrm{H}_{2} \mathrm{O}$ & 1 & $\mathrm{SO}_{4}^{-2}$ & & \\
\hline Larnakite & -10.194 & -9.941 & -2 & $\mathrm{H}^{+1}$ & 2 & $\mathrm{~Pb}^{+2}$ & 1 & $\mathrm{SO}_{4}^{-2}$ & 1 & $\mathrm{H}_{2} \mathrm{O}$ & & \\
\hline Laurionite & -7.518 & -8.141 & -1 & $\mathrm{H}^{+1}$ & 1 & $\mathrm{~Pb}^{+2}$ & 1 & $\mathrm{Cl}^{-1}$ & 1 & $\mathrm{H}_{2} \mathrm{O}$ & & \\
\hline Lepidocrocite & 5.665 & 4.294 & -3 & $\mathrm{H}^{+1}$ & 1 & $\mathrm{Fe}^{+3}$ & 2 & $\mathrm{H}_{2} \mathrm{O}$ & & & & \\
\hline Lime & 3.811 & -30.5 & -2 & $\mathrm{H}^{+1}$ & 1 & $\mathrm{Ca}^{+2}$ & 1 & $\mathrm{H}_{2} \mathrm{O}$ & & & & \\
\hline Litharge & -0.545 & -13.779 & 1 & $\mathrm{~Pb}^{+2}$ & 1 & $\mathrm{H}_{2} \mathrm{O}$ & -2 & $\mathrm{H}^{+1}$ & & & & \\
\hline Maghemite & 11.331 & 4.945 & -6 & $\mathrm{H}^{+1}$ & 2 & $\mathrm{Fe}^{+3}$ & 3 & $\mathrm{H}_{2} \mathrm{O}$ & & & & \\
\hline Magnesioferrite & 14.523 & -4.654 & -8 & $\mathrm{H}^{+1}$ & 1 & $\mathrm{Mg}^{+2}$ & 2 & $\mathrm{Fe}^{+3}$ & 4 & $\mathrm{H}_{2} \mathrm{O}$ & & \\
\hline Magnesite & -29.724 & -22.098 & 1 & $\mathrm{Mg}^{+2}$ & 1 & $\mathrm{CO}_{3}^{-2}$ & & & & & & \\
\hline Magnetite & 14.221 & 9.086 & -8 & $\mathrm{H}^{+1}$ & 2 & $\mathrm{Fe}^{+3}$ & 1 & $\mathrm{Fe}^{+2}$ & 4 & $\mathrm{H}_{2} \mathrm{O}$ & & \\
\hline Malachite & -35.913 & -30.811 & 2 & $\mathrm{Cu}^{+2}$ & 2 & $\mathrm{H}_{2} \mathrm{O}$ & -2 & $\mathrm{H}^{+1}$ & 1 & $\mathrm{CO}_{3}{ }^{-2}$ & & \\
\hline Massicot & -0.545 & -13.991 & 1 & $\mathrm{~Pb}^{+2}$ & 1 & $\mathrm{H}_{2} \mathrm{O}$ & -2 & $\mathrm{H}^{+1}$ & & & & \\
\hline Melanothallite & -15.444 & -22.228 & 1 & $\mathrm{Cu}^{+2}$ & 2 & $\mathrm{Cl}^{-1}$ & & & & & & \\
\hline Melanterite & -6.213 & -3.834 & 1 & $\mathrm{Fe}^{+2}$ & 1 & $\mathrm{SO}_{4}^{-2}$ & 7 & $\mathrm{H}_{2} \mathrm{O}$ & & & & \\
\hline $\mathrm{Mg}(\mathrm{OH})_{2}$ (active) & 3.192 & -15.602 & 1 & $\mathrm{Mg}^{+2}$ & 2 & $\mathrm{H}_{2} \mathrm{O}$ & -2 & $\mathrm{H}^{+1}$ & & & & \\
\hline $\mathrm{Mg}_{2}(\mathrm{OH})_{3} \mathrm{Cl}_{{ }_{4}} \mathrm{H}_{2} \mathrm{O}(\mathrm{s})$ & -0.588 & -26.588 & 2 & $\mathrm{Mg}^{+2}$ & 1 & $\mathrm{Cl}^{-1}$ & -3 & $\mathrm{H}^{+1}$ & 7 & $\mathrm{H}_{2} \mathrm{O}$ & & \\
\hline $\mathrm{Mg}_{3}\left(\mathrm{PO}_{4}\right)_{2}(\mathrm{~s})$ & -51.842 & -28.562 & 3 & $\mathrm{Mg}^{+2}$ & 2 & $\mathrm{PO}_{4}^{-3}$ & & & & & & \\
\hline $\mathrm{MgCO}_{3}:{ }_{5} \mathrm{H}_{2} \mathrm{O}(\mathrm{s})$ & -29.724 & -25.184 & 1 & $\mathrm{Mg}^{+2}$ & 1 & $\mathrm{CO}_{3}^{-2}$ & 5 & $\mathrm{H}_{2} \mathrm{O}$ & & & & \\
\hline $\mathrm{MgHPO}_{4}:_{3} \mathrm{H}_{2} \mathrm{O}(\mathrm{s})$ & -27.517 & -9.342 & 1 & $\mathrm{Mg}^{+2}$ & 1 & $\mathrm{H}^{+1}$ & 1 & $\mathrm{PO}_{4}^{-3}$ & 3 & $\mathrm{H}_{2} \mathrm{O}$ & & \\
\hline Mirabilite & -9.142 & -7.368 & 2 & $\mathrm{Na}^{+1}$ & 1 & $\mathrm{SO}_{4}^{-2}$ & 10 & $\mathrm{H}_{2} \mathrm{O}$ & & & & \\
\hline $\mathrm{Mn}_{3}\left(\mathrm{AsO}_{4}\right)_{2}:{ }_{8} \mathrm{H}_{2} \mathrm{O}(\mathrm{s})$ & -42.957 & -14.257 & 3 & $\mathrm{Mn}^{+2}$ & 2 & $\mathrm{AsO}_{4}^{-3}$ & 8 & $\mathrm{H}_{2} \mathrm{O}$ & & & & \\
\hline $\mathrm{Mn}_{3}\left(\mathrm{PO}_{4}\right)_{2}(\mathrm{~s})$ & -51.676 & -27.775 & 3 & $\mathrm{Mn}^{+2}$ & 2 & $\mathrm{PO}_{4}^{-3}$ & & & & & & \\
\hline $\mathrm{MnCl}_{2}: 4 \mathrm{H}_{2} \mathrm{O}(\mathrm{s})$ & -10.698 & -13.503 & 1 & $\mathrm{Mn}^{+2}$ & 2 & $\mathrm{Cl}^{-1}$ & 4 & $\mathrm{H}_{2} \mathrm{O}$ & & & & \\
\hline $\mathrm{MnCO}_{3}(\mathrm{am})$ & -29.669 & -19.169 & 1 & $\mathrm{Mn}^{+2}$ & 1 & $\mathrm{CO}_{3}^{-2}$ & & & & & & \\
\hline $\mathrm{MnHPO}_{4}(\mathrm{~s})$ & -27.462 & -2.062 & 1 & $\mathrm{Mn}^{+2}$ & 1 & $\mathrm{PO}_{4}^{-3}$ & 1 & $\mathrm{H}^{+1}$ & & & & \\
\hline $\mathrm{MnSO}_{4}(\mathrm{~s})$ & -5.857 & -8.978 & 1 & $\mathrm{Mn}^{+2}$ & 1 & $\mathrm{SO}_{4}^{-2}$ & & & & & & \\
\hline Na-Jarosite & -1.231 & 2.961 & 1 & $\mathrm{Na}^{+1}$ & 3 & $\mathrm{Fe}^{+3}$ & 2 & $\mathrm{SO}_{4}^{-2}$ & -6 & $\mathrm{H}^{+1}$ & 6 & $\mathrm{H}_{2} \mathrm{O}$ \\
\hline Natron & -32.954 & -31.096 & 2 & $\mathrm{Na}^{+1}$ & 1 & $\mathrm{CO}_{3}^{-2}$ & 10 & $\mathrm{H}_{2} \mathrm{O}$ & & & & \\
\hline Nesquehonite & -29.724 & -25.255 & 1 & $\mathrm{Mg}^{+2}$ & 1 & $\mathrm{CO}_{3}^{-2}$ & 3 & $\mathrm{H}_{2} \mathrm{O}$ & & & & \\
\hline Otavite & -33.13 & -21.063 & 1 & $\mathrm{Cd}^{+2}$ & 1 & $\mathrm{CO}_{3}^{-2}$ & & & & & & \\
\hline $\mathrm{Pb}(\mathrm{OH})_{2}(\mathrm{~s})$ & -0.545 & -9.181 & -2 & $\mathrm{H}^{+1}$ & 1 & $\mathrm{~Pb}^{+2}$ & 2 & $\mathrm{H}_{2} \mathrm{O}$ & & & & \\
\hline $\mathrm{Pb}_{10}(\mathrm{OH})_{6} \mathrm{O}\left(\mathrm{CO}_{3}\right)_{6}(\mathrm{~s})$ & -202.947 & -194.187 & 10 & $\mathrm{~Pb}^{+2}$ & 6 & $\mathrm{CO}_{3}^{-2}$ & 7 & $\mathrm{H}_{2} \mathrm{O}$ & -8 & $\mathrm{H}^{+1}$ & & \\
\hline $\mathrm{Pb}_{2}(\mathrm{OH})_{3} \mathrm{Cl}(\mathrm{s})$ & -8.063 & -16.856 & -3 & $\mathrm{H}^{+1}$ & 2 & $\mathrm{~Pb}^{+2}$ & 3 & $\mathrm{H}_{2} \mathrm{O}$ & 1 & $\mathrm{Cl}^{-1}$ & & \\
\hline $\mathrm{Pb}_{2} \mathrm{O}(\mathrm{OH})_{2}(\mathrm{~s})$ & -1.09 & -27.28 & 2 & $\mathrm{~Pb}^{+2}$ & 3 & $\mathrm{H}_{2} \mathrm{O}$ & -4 & $\mathrm{H}^{+1}$ & & & & \\
\hline $\mathrm{Pb}_{2} \mathrm{OCO}_{3}(\mathrm{~s})$ & -34.006 & -33.788 & -2 & $\mathrm{H}^{+1}$ & 2 & $\mathrm{~Pb}^{+2}$ & 1 & $\mathrm{H}_{2} \mathrm{O}$ & 1 & $\mathrm{CO}_{3}^{-2}$ & & \\
\hline $\mathrm{Pb}_{3}\left(\mathrm{AsO}_{4}\right)_{2}(\mathrm{~s})$ & -54.335 & -18.835 & 3 & $\mathrm{~Pb}^{+2}$ & 2 & $\mathrm{AsO}_{4}^{-3}$ & & & & & & \\
\hline $\mathrm{Pb}_{3}\left(\mathrm{PO}_{4}\right)_{2}(\mathrm{~s})$ & -63.053 & -19.523 & 3 & $\mathrm{~Pb}^{+2}$ & 2 & $\mathrm{PO}_{4}^{-3}$ & & & & & & \\
\hline $\mathrm{Pb}_{3} \mathrm{O}_{2} \mathrm{CO}_{3}(\mathrm{~s})$ & -34.551 & -46.49 & -4 & $\mathrm{H}^{+1}$ & 3 & $\mathrm{~Pb}^{+2}$ & 1 & $\mathrm{CO}_{3}^{-2}$ & 2 & $\mathrm{H}_{2} \mathrm{O}$ & & \\
\hline $\mathrm{Pb}_{3} \mathrm{O}_{2} \mathrm{SO}_{4}(\mathrm{~s})$ & -10.739 & -22.083 & -4 & $\mathrm{H}^{+1}$ & 3 & $\mathrm{~Pb}^{+2}$ & 1 & $\mathrm{SO}_{4}^{-2}$ & 2 & $\mathrm{H}_{2} \mathrm{O}$ & & \\
\hline $\mathrm{Pb}_{4}(\mathrm{OH})_{6} \mathrm{SO}_{4}(\mathrm{~s})$ & -11.285 & -32.385 & -6 & $\mathrm{H}^{+1}$ & 4 & $\mathrm{~Pb}^{+2}$ & 1 & $\mathrm{SO}_{4}^{-2}$ & 6 & $\mathrm{H}_{2} \mathrm{O}$ & & \\
\hline
\end{tabular}


Table 4 (continued)

\begin{tabular}{|c|c|c|c|c|c|c|c|c|c|c|c|c|}
\hline \multirow{2}{*}{$\begin{array}{l}\text { Mineral } \\
\mathrm{Pb}_{4} \mathrm{O}_{3} \mathrm{SO}_{4}(\mathrm{~s})\end{array}$} & \multirow{2}{*}{$\frac{\log \text { IAP }}{-11.285}$} & \multirow{2}{*}{$\frac{\text { Sat. index }}{-34.295}$} & \multicolumn{10}{|c|}{ Stoichiometry } \\
\hline & & & -6 & $\mathrm{H}^{+1}$ & 4 & $\mathrm{~Pb}^{+2}$ & 1 & $\mathrm{SO}_{4}{ }^{-2}$ & 3 & $\mathrm{H}_{2} \mathrm{O}$ & & \\
\hline $\mathrm{PbHPO}_{4}(\mathrm{~s})$ & -31.254 & -7.449 & 1 & $\mathrm{~Pb}^{+2}$ & 1 & $\mathrm{H}^{+1}$ & 1 & $\mathrm{PO}_{4}^{-3}$ & & & & \\
\hline $\mathrm{PbO}_{0 \cdot 0} \mathrm{H}_{2} \mathrm{O}(\mathrm{s})$ & -0.545 & -13.525 & -2 & $\mathrm{H}^{+1}$ & 1 & $\mathrm{~Pb}^{+2}$ & 1.33 & $\mathrm{H}_{2} \mathrm{O}$ & & & & \\
\hline Periclase & 3.192 & -19.649 & -2 & $\mathrm{H}^{+1}$ & 1 & $\mathrm{Mg}^{+2}$ & 1 & $\mathrm{H}_{2} \mathrm{O}$ & & & & \\
\hline Phosgenite & -47.951 & -28.141 & 2 & $\mathrm{~Pb}^{+2}$ & 2 & $\mathrm{Cl}^{-1}$ & 1 & $\mathrm{CO}_{3}^{-2}$ & & & & \\
\hline Plumbgummite & -53.263 & -20.473 & -5 & $\mathrm{H}^{+1}$ & 1 & $\mathrm{~Pb}^{+2}$ & 3 & $\mathrm{Al}^{+3}$ & 2 & $\mathrm{PO}_{4}^{-3}$ & 6 & $\mathrm{H}_{2} \mathrm{O}$ \\
\hline Portlandite & 3.811 & -19.962 & 1 & $\mathrm{Ca}^{+2}$ & 2 & $\mathrm{H}_{2} \mathrm{O}$ & -2 & $\mathrm{H}^{+1}$ & & & & \\
\hline Pyrochroite & 3.247 & -12.753 & 1 & $\mathrm{Mn}^{+2}$ & 2 & $\mathrm{H}_{2} \mathrm{O}$ & -2 & $\mathrm{H}^{+1}$ & & & & \\
\hline Quartz & -3.465 & 0.721 & 1 & $\mathrm{H}_{4} \mathrm{SiO}_{4}$ & -2 & $\mathrm{H}_{2} \mathrm{O}$ & & & & & & \\
\hline Rhodochrosite & -29.669 & -18.684 & 1 & $\mathrm{Mn}^{+2}$ & 1 & $\mathrm{CO}_{3}^{-2}$ & & & & & & \\
\hline Sepiolite & -4.011 & -20.719 & 2 & $\mathrm{Mg}^{+2}$ & 3 & $\mathrm{H}_{4} \mathrm{SiO}_{4}$ & -4 & $\mathrm{H}^{+1}$ & -0.5 & $\mathrm{H}_{2} \mathrm{O}$ & & \\
\hline Sepiolite (A) & -4.011 & -22.791 & -0.5 & $\mathrm{H}_{2} \mathrm{O}$ & 2 & $\mathrm{Mg}^{+2}$ & 3 & $\mathrm{H}_{4} \mathrm{SiO}_{4}$ & -4 & $\mathrm{H}^{+1}$ & & \\
\hline Siderite & -30.025 & -19.496 & 1 & $\mathrm{Fe}^{+2}$ & 1 & $\mathrm{CO}_{3}^{-2}$ & & & & & & \\
\hline $\mathrm{SiO}_{2}(\mathrm{am}, \mathrm{gel})$ & -3.465 & -0.639 & 1 & $\mathrm{H}_{4} \mathrm{SiO}_{4}$ & -2 & $\mathrm{H}_{2} \mathrm{O}$ & & & & & & \\
\hline $\mathrm{SiO}_{2}(\mathrm{am}, \mathrm{ppt})$ & -3.465 & -0.599 & 1 & $\mathrm{H}_{4} \mathrm{SiO}_{4}$ & -2 & $\mathrm{H}_{2} \mathrm{O}$ & & & & & & \\
\hline Smithsonite & -29.71 & -18.841 & 1 & $\mathrm{Zn}^{+2}$ & 1 & $\mathrm{CO}_{3}^{-2}$ & & & & & & \\
\hline Spinel & 8.992 & -31.079 & -8 & $\mathrm{H}^{+1}$ & 1 & $\mathrm{Mg}^{+2}$ & 2 & $\mathrm{Al}^{+3}$ & 4 & $\mathrm{H}_{2} \mathrm{O}$ & & \\
\hline $\mathrm{SrHPO}_{4}(\mathrm{~s})$ & -29.447 & -10.152 & 1 & $\mathrm{Sr}^{+2}$ & 1 & $\mathrm{H}^{+1}$ & 1 & $\mathrm{PO}_{4}^{-3}$ & & & & \\
\hline Strengite & -25.044 & 1.279 & 1 & $\mathrm{Fe}^{+3}$ & 1 & $\mathrm{PO}_{4}^{-3}$ & 2 & $\mathrm{H}_{2} \mathrm{O}$ & & & & \\
\hline Strontianite & -31.654 & -22.392 & 1 & $\mathrm{Sr}^{+2}$ & 1 & $\mathrm{CO}_{3}^{-2}$ & & & & & & \\
\hline Tenorite(am) & -1.499 & -10.528 & 1 & $\mathrm{Cu}^{+2}$ & 1 & $\mathrm{H}_{2} \mathrm{O}$ & -2 & $\mathrm{H}^{+1}$ & & & & \\
\hline Tenorite(c) & -1.499 & -9.678 & 1 & $\mathrm{Cu}^{+2}$ & -2 & $\mathrm{H}^{+1}$ & 1 & $\mathrm{H}_{2} \mathrm{O}$ & & & & \\
\hline Thenardite & -9.142 & -9.54 & 2 & $\mathrm{Na}^{+1}$ & 1 & $\mathrm{SO}_{4}^{-2}$ & & & & & & \\
\hline Thermonatrite & -32.954 & -33.678 & 2 & $\mathrm{Na}^{+1}$ & 1 & $\mathrm{CO}_{3}^{-2}$ & 1 & $\mathrm{H}_{2} \mathrm{O}$ & & & & \\
\hline Tsumebite & -33.298 & -23.508 & -3 & $\mathrm{H}^{+1}$ & 2 & $\mathrm{~Pb}^{+2}$ & 1 & $\mathrm{Cu}^{+2}$ & 1 & $\mathrm{PO}_{4}^{-3}$ & 6 & $\mathrm{H}_{2} \mathrm{O}$ \\
\hline Vaterite & -29.105 & -21.293 & 1 & $\mathrm{Ca}^{+2}$ & 1 & $\mathrm{CO}_{3}^{-2}$ & & & & & & \\
\hline Vivianite & -52.746 & -14.944 & 3 & $\mathrm{Fe}^{+2}$ & 2 & $\mathrm{PO}_{4}^{-3}$ & 8 & $\mathrm{H}_{2} \mathrm{O}$ & & & & \\
\hline Zincite & 3.206 & -8.768 & 1 & $\mathrm{Zn}^{+2}$ & 1 & $\mathrm{H}_{2} \mathrm{O}$ & -2 & $\mathrm{H}^{+1}$ & & & & \\
\hline Zincosite & -5.898 & -10.514 & 1 & $\mathrm{Zn}^{+2}$ & 1 & $\mathrm{SO}_{4}^{-2}$ & & & & & & \\
\hline $\mathrm{Zn}\left(\mathrm{NO}_{3}\right)_{2}:{ }_{6} \mathrm{H}_{2} \mathrm{O}(\mathrm{s})$ & -16.586 & -19.697 & 1 & $\mathrm{Zn}^{+2}$ & 2 & $\mathrm{NO}_{3}^{-1}$ & 6 & $\mathrm{H}_{2} \mathrm{O}$ & & & & \\
\hline $\mathrm{Zn}(\mathrm{OH})_{2}(\mathrm{am})$ & 3.206 & -9.979 & 1 & $\mathrm{Zn}^{+2}$ & 2 & $\mathrm{H}_{2} \mathrm{O}$ & -2 & $\mathrm{H}^{+1}$ & & & & \\
\hline $\mathrm{Zn}(\mathrm{OH})_{2}$ (beta) & 3.206 & -9.239 & 1 & $\mathrm{Zn}^{+2}$ & 2 & $\mathrm{H}_{2} \mathrm{O}$ & -2 & $\mathrm{H}^{+1}$ & & & & \\
\hline $\mathrm{Zn}(\mathrm{OH})_{2}$ (delta) & 3.206 & -8.638 & 1 & $\mathrm{Zn}^{+2}$ & -2 & $\mathrm{H}^{+1}$ & 2 & $\mathrm{H}_{2} \mathrm{O}$ & & & & \\
\hline $\mathrm{Zn}(\mathrm{OH})_{2}$ (epsilon) & 3.206 & -8.981 & 1 & $\mathrm{Zn}^{+2}$ & 2 & $\mathrm{H}_{2} \mathrm{O}$ & -2 & $\mathrm{H}^{+1}$ & & & & \\
\hline $\mathrm{Zn}(\mathrm{OH})_{2}$ (gamma) & 3.206 & -9.208 & 1 & $\mathrm{Zn}^{+2}$ & 2 & $\mathrm{H}_{2} \mathrm{O}$ & -2 & $\mathrm{H}^{+1}$ & & & & \\
\hline $\mathrm{Zn}_{2}(\mathrm{OH})_{2} \mathrm{SO}_{4}(\mathrm{~s})$ & -2.692 & -10.192 & -2 & $\mathrm{H}^{+1}$ & 2 & $\mathrm{Zn}^{+2}$ & 2 & $\mathrm{H}_{2} \mathrm{O}$ & 1 & $\mathrm{SO}_{4}^{-2}$ & & \\
\hline $\mathrm{Zn}_{2}(\mathrm{OH})_{3} \mathrm{Cl}(\mathrm{s})$ & -0.56 & -15.751 & 2 & $\mathrm{Zn}^{+2}$ & 3 & $\mathrm{H}_{2} \mathrm{O}$ & -3 & $\mathrm{H}^{+1}$ & 1 & $\mathrm{Cl}^{-1}$ & & \\
\hline $\mathrm{Zn}_{3}\left(\mathrm{PO}_{4}\right)_{2}:{ }_{4} \mathrm{H}_{2} \mathrm{O}(\mathrm{s})$ & -51.8 & -16.38 & 3 & $\mathrm{Zn}^{+2}$ & 2 & $\mathrm{PO}_{4}^{-3}$ & 4 & $\mathrm{H}_{2} \mathrm{O}$ & & & & \\
\hline $\mathrm{Zn}_{3} \mathrm{AsO}_{42} \cdot 2 \cdot 5 \mathrm{H}_{2} \mathrm{O}(\mathrm{s})$ & -43.081 & -15.581 & 3 & $\mathrm{Zn}^{+2}$ & 2 & $\mathrm{AsO}_{4}^{-3}$ & 2.5 & $\mathrm{H}_{2} \mathrm{O}$ & & & & \\
\hline $\mathrm{Zn}_{3} \mathrm{O}\left(\mathrm{SO}_{4}\right)_{2}(\mathrm{~s})$ & -8.59 & -29.647 & -2 & $\mathrm{H}^{+1}$ & 3 & $\mathrm{Zn}^{+2}$ & 2 & $\mathrm{SO}_{4}^{-2}$ & 1 & $\mathrm{H}_{2} \mathrm{O}$ & & \\
\hline $\mathrm{Zn}_{4}(\mathrm{OH})_{6} \mathrm{SO}_{4}(\mathrm{~s})$ & 3.721 & -24.679 & -6 & $\mathrm{H}^{+1}$ & 4 & $\mathrm{Zn}^{+2}$ & 6 & $\mathrm{H}_{2} \mathrm{O}$ & 1 & $\mathrm{SO}_{4}^{-2}$ & & \\
\hline $\mathrm{Zn}_{5}(\mathrm{OH})_{8} \mathrm{Cl}_{2}(\mathrm{~s})$ & 2.086 & -36.414 & -8 & $\mathrm{H}^{+1}$ & 5 & $\mathrm{Zn}^{+2}$ & 8 & $\mathrm{H}_{2} \mathrm{O}$ & 2 & $\mathrm{Cl}^{-1}$ & & \\
\hline Zn-Al LDH(s) & -7.146 & -26.976 & 2 & $\mathrm{Zn}^{+2}$ & 1 & $\mathrm{Al}^{+3}$ & 0.5 & $\mathrm{CO}_{3}^{-2}$ & -6 & $\mathrm{H}^{+1}$ & 6 & $\mathrm{H}_{2} \mathrm{O}$ \\
\hline $\mathrm{ZnCl}_{2}(\mathrm{~s})$ & -10.739 & -18.391 & 1 & $\mathrm{Zn}^{+2}$ & 2 & $\mathrm{Cl}^{-1}$ & & & & & & \\
\hline
\end{tabular}


Table 4 (continued)

\begin{tabular}{lllllllll}
\hline Mineral & Log IAP & Sat. index & Stoichiometry & & & & \\
\hline $\mathrm{ZnCO}_{3}(\mathrm{~s})$ & -29.71 & -18.91 & 1 & $\mathrm{Zn}^{+2}$ & 1 & $\mathrm{CO}_{3}{ }^{-2}$ & & \\
$\mathrm{ZnCO}_{3}: \mathrm{H}_{2} \mathrm{O}(\mathrm{s})$ & -29.71 & -19.45 & 1 & $\mathrm{Zn}^{+2}$ & 1 & $\mathrm{CO}_{3}{ }^{-2}$ & 1 & $\mathrm{H}_{2} \mathrm{O}$ \\
$\mathrm{ZnSO}_{4}: \mathrm{H}_{2} \mathrm{O}(\mathrm{s})$ & -5.898 & -5.626 & 1 & $\mathrm{Zn}^{+2}$ & 1 & $\mathrm{SO}_{4}^{-2}$ & 1 & $\mathrm{H}_{2} \mathrm{O}$ \\
\hline
\end{tabular}

of accessory minerals (e.g. Ti minerals) and perhaps traces of sulphidic ores. Lastly, $\mathrm{C}$ and $\mathrm{N}$ contents are very low, emphasizing the mineral nature of the sediment and the truly very minor contribution of microorganisms to the sediment contained in the terrace rims.

Though sediments from the various layers in the moss section (samples P3-7) were not fully analysed (e.g. no XRF data), the data presented in Table 3 demonstrate that over time variations in composition are limited and rather invariable schwertmannite-type material formed an important component of the material, trapped as sediment by or precipitated on the moss. The same holds for As fixation/sorption.

\subsection{Moss and Its Coatings}

Quite informative are the data in Table 2. Excluding those elements that are evidently contained in the living plant material (e.g. $\mathrm{K}, \mathrm{Mg}$ and $\mathrm{P}$ ), two types of coatings can be distinguished: thin translucent early coatings, largely composed of $\mathrm{Fe}$ and $\mathrm{Al}$ hydroxides with minor amounts of As and very low S contents, and later dense creamy coatings, which in addition to these hydroxides contain S and As. Moreover, in this subsequent stage, detrital material is incorporated and the composition is closer to that of the amorphous component of the sediment. It should be emphasized that these observations concern the 'early precipitates' since all green moss samples were thoroughly washed to remove fine interstitial material that did not adhere to this moss. Results for the latter material (P1-P2) evidence that this material is much lower in $\mathrm{Al}$ and contains less $\mathrm{S}$, whereas the fixation/sorption of As seemingly has continued to reach rather extreme values (molar ratios of $\mathrm{As} /(\mathrm{As}+\mathrm{S})$ are between 0.18 and 0.22 ), which is not yet the maximum ratio for As sorption on schwertmannite to become unstable (Carlson et al. 2002; Fukushi et al. 2003).

At first sight, puzzling are the relatively high $\mathrm{Al}$ contents $(\mathrm{Al} / \mathrm{Fe}$ molar ratios are about $1.2-1.6)$ of the early precipitates (see Table 2), since in the amorphous component of the sediment $\mathrm{Al}$ contents are much lower (see Table 3). We attribute this to preferential adsorption of the trivalent $\mathrm{Al}^{3+}$ ion (and $\mathrm{Fe}^{3+}$ ) by the living moss that has a high cation exchange capacity, implying that upon its senescence and death, such adsorption capacity disappears and precipitation of schwertmannite-type material and As fixation/sorption prevails.

\subsection{Stream Water Chemistry}

In 2008, Loayza-Muro et al. (2010) sampled stream water at the same location over four seasons and in triplicate. Water samples were not filtered and for estimation of metals were acidified with $10 \mathrm{M} \mathrm{HNO}_{3}$ prior to their analysis by ICP-OES. Their results thus included metals that were present as acid-soluble fine suspended material and cannot be compared with our results. Moreover, S content was not established, for which reason a check on their data based on the electroneutrality principle is impossible (see also Section 4.2). For details on the methods used, reference is made to Loayza-Muro et al. (2010). Over the past years, the mines and ore treatment plants operated intermittently and discharge of acid mine drainage varied over the years. This may explain the significant differences between the 2008 data and our 2010 data, but we cannot exclude analytical errors, since $\mathrm{pH}$ and $\mathrm{EC}$ values are similar to our values, but electrolyte contents are distinctly lower, which is hard to explain.

Based on our 2010 data, ion and ion pair concentrations were calculated, and ion activity products were compared with equilibrium constants of various relevant minerals (data not presented). Saturation indices indicate that potentially schwertmannite, tooeleite (both might contain arsenic) and jarosite might precipitate. Jarosite can be detected by X-ray analysis, but as already stated was not observed. This leaves schwertmannite/ tooeleite (the 'schwertmannite-type' material) as the main precipitate to be formed in the stream on the basis of our water data, in conformance with our actual observations. 
Table 5 Component and percent of total concentration

\begin{tabular}{|c|c|c|c|c|c|}
\hline Component & $\%$ of total concentration & Species name & Component & $\%$ of total concentration & Species name \\
\hline \multirow[t]{2}{*}{$\mathrm{CO}_{3}{ }^{-2}$} & 0.084 & $\mathrm{HCO}_{3-}$ & & 1.613 & $\mathrm{FeH}_{2} \mathrm{PO}_{4}^{+2}$ \\
\hline & 99.913 & $\mathrm{H}_{2} \mathrm{CO}_{3}(\mathrm{aq})$ & & 96.921 & $\mathrm{FeHPO}_{4+}$ \\
\hline \multirow[t]{2}{*}{$\mathrm{K}^{+1}$} & 98.287 & $\mathrm{~K}_{+1}$ & & 0.037 & $\mathrm{CaH}_{2} \mathrm{PO}_{4+}$ \\
\hline & 1.704 & $\mathrm{KSO}_{4-}$ & $\mathrm{SO}_{4}^{-2}$ & 56.691 & $\mathrm{SO}_{4}^{-2}$ \\
\hline \multirow[t]{3}{*}{$\mathrm{Na}^{+1}$} & 98.329 & $\mathrm{Na}_{+1}$ & & 1.187 & $\mathrm{HSO}_{4-}$ \\
\hline & 0.01 & $\mathrm{NaCl}(\mathrm{aq})$ & & 0.018 & $\mathrm{AlSO}_{4+}$ \\
\hline & 1.661 & $\mathrm{NaSO}_{4-}$ & & 2.318 & $\mathrm{ZnSO}_{4}(\mathrm{aq})$ \\
\hline \multirow[t]{3}{*}{$\mathrm{Ca}^{+2}$} & 75.365 & $\mathrm{Ca}_{+2}$ & & 0.273 & $\mathrm{Zn}\left(\mathrm{SO}_{4}\right)_{2}{ }^{-2}$ \\
\hline & 0.023 & $\mathrm{CaCl}+$ & & 1.215 & $\mathrm{FeSO}_{4}(\mathrm{aq})$ \\
\hline & 24.612 & $\mathrm{CaSO}_{4}(\mathrm{aq})$ & & 22.449 & $\mathrm{FeSO}_{4+}$ \\
\hline \multirow[t]{3}{*}{$\mathrm{Mg}^{+2}$} & 78.963 & $\mathrm{Mg}_{+2}$ & & 2.12 & $\mathrm{Fe}\left(\mathrm{SO}_{4}\right)_{2-}$ \\
\hline & 0.038 & $\mathrm{MgCl}+$ & & 1.975 & $\mathrm{MnSO}_{4}(\mathrm{aq})$ \\
\hline & 20.999 & $\mathrm{MgSO}_{4}(\mathrm{aq})$ & & 1.881 & $\mathrm{MgSO}_{4}(\mathrm{aq})$ \\
\hline \multirow[t]{4}{*}{$\mathrm{Al}^{+3}$} & 16.406 & $\mathrm{Al}_{+3}$ & & 9.599 & $\mathrm{CaSO}_{4}(\mathrm{aq})$ \\
\hline & 0.059 & $\mathrm{AlOH}_{+2}$ & & 0.023 & $\mathrm{SrSO}_{4}(\mathrm{aq})$ \\
\hline & 74.492 & $\mathrm{AlSO}_{4+}$ & & 0.09 & $\mathrm{NaSO}_{4-}$ \\
\hline & 9.036 & $\mathrm{Al}\left(\mathrm{SO}_{4}\right)_{2-}$ & & 0.018 & $\mathrm{KSO}_{4-}$ \\
\hline \multirow[t]{3}{*}{$\mathrm{Mn}^{+2}$} & 80.258 & $\mathrm{Mn}_{+2}$ & & 0.128 & $\mathrm{NH}_{4} \mathrm{SO}_{4-}$ \\
\hline & 0.011 & $\mathrm{MnCl}+$ & $\mathrm{H}_{4} \mathrm{SiO}_{4}$ & 99.833 & $\mathrm{H}_{4} \mathrm{SiO}_{4}$ \\
\hline & 19.732 & $\mathrm{MnSO}_{4}(\mathrm{aq})$ & & 0.167 & $\mathrm{H}_{4} \mathrm{SiO}_{4} \mathrm{SO}_{4}^{-2}$ \\
\hline \multirow[t]{4}{*}{$\mathrm{Zn}^{+2}$} & 74.832 & $\mathrm{Zn}_{+2}$ & $\mathrm{NH}_{4}^{+1}$ & 96.752 & $\mathrm{NH}_{4}^{+1}$ \\
\hline & 0.026 & $\mathrm{ZnCl}+$ & & 3.248 & $\mathrm{NH}_{4} \mathrm{SO}_{4-}$ \\
\hline & 23.743 & $\mathrm{ZnSO}_{4}(\mathrm{aq})$ & $\mathrm{NO}_{3}^{-1}$ & 99.235 & $\mathrm{NO}_{3}^{-1}$ \\
\hline & 1.4 & $\mathrm{Zn}\left(\mathrm{SO}_{4}\right)_{2}{ }^{-2}$ & & 0.11 & $\mathrm{ZnNO}_{3+}$ \\
\hline \multirow[t]{4}{*}{$\mathrm{Pb}^{+2}$} & 54.562 & $\mathrm{~Pb}_{+2}$ & & 0.07 & $\mathrm{MnNO}_{3+}$ \\
\hline & 0.22 & $\mathrm{PbCl}+$ & & 0.565 & $\mathrm{CaNO}_{3+}$ \\
\hline & 43.636 & $\mathrm{PbSO}_{4}(\mathrm{aq})$ & & 0.011 & $\mathrm{NaNO}_{3}$ (aq) \\
\hline & 1.581 & $\mathrm{~Pb}\left(\mathrm{SO}_{4}\right)_{2}{ }^{-2}$ & $\mathrm{Cl}^{-1}$ & 99.11 & $\mathrm{Cl}^{-1}$ \\
\hline \multirow[t]{3}{*}{$\mathrm{AsO}_{4}{ }^{-3}$} & 6.763 & $\mathrm{H}_{3} \mathrm{AsO}_{4}$ & & 0.104 & $\mathrm{ZnCl}+$ \\
\hline & 0.029 & $\mathrm{HAsO}_{4}^{-2}$ & & 0.375 & $\mathrm{CaCl}+$ \\
\hline & 93.207 & $\mathrm{H}_{2} \mathrm{AsO}_{4-}$ & & 0.182 & $\mathrm{FeCl}^{+2}$ \\
\hline \multirow[t]{4}{*}{$\mathrm{Cd}^{+2}$} & 73.142 & $\mathrm{Cd}_{+2}$ & & 0.044 & $\mathrm{MnCl}+$ \\
\hline & 0.881 & $\mathrm{CdCl}+$ & & 0.012 & $\mathrm{FeCl}+$ \\
\hline & 23.705 & $\mathrm{CdSO}_{4}(\mathrm{aq})$ & & 0.143 & $\mathrm{MgCl}+$ \\
\hline & 2.271 & $\mathrm{Cd}\left(\mathrm{SO}_{4}\right)_{2}{ }^{-2}$ & & 0.023 & $\mathrm{NaCl}(\mathrm{aq})$ \\
\hline \multirow[t]{4}{*}{$\mathrm{Cu}^{+2}$} & 75.92 & $\mathrm{Cu}_{+2}$ & $\mathrm{Fe}^{+3}$ & 5.518 & $\mathrm{Fe}^{+3}$ \\
\hline & 0.017 & $\mathrm{CuCl}+$ & & 29.058 & $\mathrm{FeOH}^{+2}$ \\
\hline & 24.045 & $\mathrm{CuSO}_{4}(\mathrm{aq})$ & & 5.231 & $\mathrm{Fe}(\mathrm{OH})_{2+}$ \\
\hline & 0.016 & $\mathrm{CuHSO}_{4+}$ & & 3.43 & $\mathrm{Fe}_{2}(\mathrm{OH})_{2}^{+4}$ \\
\hline \multirow[t]{3}{*}{$\mathrm{Sr}^{+2}$} & 78.14 & $\mathrm{Sr}_{+2}$ & & 1.735 & $\mathrm{Fe}_{3}(\mathrm{OH})_{4}^{+5}$ \\
\hline & 0.014 & $\mathrm{SrCl}+$ & & 0.01 & $\mathrm{FeCl}^{+2}$ \\
\hline & 21.846 & $\mathrm{SrSO}_{4}(\mathrm{aq})$ & & 52.496 & $\mathrm{FeSO}_{4+}$ \\
\hline \multirow[t]{2}{*}{$\mathrm{PO}_{4}^{-3}$} & 1.273 & $\mathrm{H}_{2} \mathrm{PO}_{4-}$ & & 2.479 & $\mathrm{Fe}\left(\mathrm{SO}_{4}\right)_{2-}$ \\
\hline & 0.066 & $\mathrm{H}_{3} \mathrm{PO}_{4}$ & & 0.041 & $\mathrm{FeHPO}_{4+}$ \\
\hline
\end{tabular}


Table 5 (continued)

\begin{tabular}{llllll}
\hline Component & \% of total concentration & Species name & Component & \% of total concentration & Species name \\
\hline & 0.088 & $\mathrm{FeH}_{2} \mathrm{PO}_{4+}$ & $\mathrm{Fe}^{+2}$ & 74.403 & $\mathrm{Fe}^{+2}$ \\
$\mathrm{FeSO}_{4}(\mathrm{aq})$ \\
\hline
\end{tabular}

We only sampled once at the end of the dry season, and thus, our chemical stream water data are by their own no reliable estimate for the longer-term stream water composition. Much more decisive information on this composition can be deduced from our data on the chemical composition of the sediment captured in the moss rim (samples P3-7, Table 3) and the layered structure of this rim.

Seasonal variations in growth have been reported for many bryophytes, including Bryum species (e.g. Schwarz et al. 1992; Núñez-Olivera et al. 2010) and many aquatic or semi-aquatic mosses (e.g. Ilyashuk 2002; Guo et al. 2013). Assuming that the observed layering is indicative for seasonal variations in growth and environmental conditions (dry and wet season), a rough estimate of the growth rate of the moss rims can be obtained, being in the order of $4-5 \mathrm{~mm} /$ year (see also Fig. 3). This is well in accordance with growth rates observed in similar extreme environments, such as for Bryum pseudotriquetrum in continental Antarctica (Selkirk and Skotnicki 2007) and for mosses in Arctic lakes and streams (e.g. Sand-Jensen et al. 1999). Moreover, accumulation rates in Bryophyte dominated mires and peats in the high-altitude Peruvian Puna peatlands were found to be in the order of $2 \mathrm{~mm} /$ year (Salvador et al. 2014/15). Given the number of layers encountered in the highest terrace rims (up to 30), the age of the terrace rims would be in the order of at least several decades, with a growth rate of around $5 \mathrm{~mm} /$ year, which seems a realistic estimate for this truly stressed semi-aquatic environment.

Taking into account that during the dry season concentrations of solutes will be highest and schwertmannite-type material may precipitate, we can conclude that at least over several decades dry season stream water composition met the conditions required for such precipitation. España et al. (2005) and España (2007) described these as oxygenated, a pH between 2 and 4 and very high $\mathrm{Fe}^{3+}$ concentrations in the form of ferric sulphate complexes. This is clearly in line with our observed stream water composition.

\subsection{The Moss Microterraces}

Terrace growth forms are not uncommon for bryophyte species, such as Nardia compressa and Scapania paludosa. In Alaska, these species formed 30-50-cmwide terraces in a small stream, which impounded the swiftly flowing rivulet in a series of small pools (Shacklette 1965). This study by Shacklette (1965), however, remains one of the few studies describing terrace forming mosses from acid aquatic environments, whereas they are quite common in calcareous streams and springs: travertine terrace walls are often partly formed by bryophytes such as Palustriella commutata (e.g. Lang and Lucas 1970; Pentacost and Zhaohui 2002; Pentacost 2005).

España et al. (2007) reported 'iron terraces' from acid mine drainage systems, but their formation is ascribed to microbial activity and not to bryophytes. Evidently within our dense moss-built terrace rims, microbial activity may play a role since oxygen levels will be much lower than in the turbulent water. However, we did not find any indication for such activity to play a visible role in the formation of the terraces: the rims are dominantly composed of moss and its necromass, and the formation of the encrustations and coatings clearly is a secondary process, nor did we observe microstructures indicative for such microbial activity, as for example described by España et al. (2007).

It is not clear whether the terrace growth form is typical for $A$. prostratum since more detailed descriptions of its habitat do not exist. A. prostratum is an acrocarpic species without feathery branches, but it does have small leaves and a tufted growth form, which may increase encrustation. In our case, the encrustations are definitely not by calcite nor can photosynthesis be expected to play a serious role in their formation. Encrustation is rather a consequence of the successive formation of precipitates from supersaturated stream water, combined with 'capture' of fine detrital minerals. 
Table 6 Concentration and activity

\begin{tabular}{|c|c|c|c|c|c|c|c|}
\hline Species & Concentration & Activity & Log activity & Species & Concentration & Activity & Log activity \\
\hline $\mathrm{Al}(\mathrm{OH})_{2+}$ & $2.2817 \mathrm{E}-12$ & $1.9421 \mathrm{E}-12$ & -11.712 & $\mathrm{HPO}_{4}{ }^{-2}$ & $4.6841 \mathrm{E}-12$ & $2.4585 \mathrm{E}-12$ & -11.609 \\
\hline $\mathrm{Al}(\mathrm{OH})_{3}(\mathrm{aq})$ & $5.5105 \mathrm{E}-16$ & $5.5497 \mathrm{E}-16$ & -15.256 & $\mathrm{HSO}_{4-}$ & 0.00012503 & 0.00010642 & -3.973 \\
\hline $\mathrm{Al}(\mathrm{OH})_{4-}$ & $5.6181 \mathrm{E}-19$ & $4.7819 \mathrm{E}-19$ & -18.32 & $\mathrm{~K}^{+1}$ & 0.00011205 & 0.00009537 & -4.021 \\
\hline $\mathrm{Al}\left(\mathrm{SO}_{4}\right)_{2-}$ & $2.3492 \mathrm{E}-07$ & $1.9996 \mathrm{E}-07$ & -6.699 & $\mathrm{~K}_{2} \mathrm{HPO}_{4}(\mathrm{aq})$ & $2.2222 \mathrm{E}-19$ & $2.238 \mathrm{E}-19$ & -18.65 \\
\hline $\mathrm{Al}^{+3}$ & $4.2656 \mathrm{E}-07$ & $1.0002 \mathrm{E}-07$ & -7 & $\mathrm{~K}_{2} \mathrm{PO}_{4-}$ & $3.019 \mathrm{E}-27$ & $2.5696 \mathrm{E}-27$ & -26.59 \\
\hline $\mathrm{Al}_{2}(\mathrm{OH})_{2}^{+4}$ & $2.5468 \mathrm{E}-15$ & $1.9328 \mathrm{E}-16$ & -15.714 & $\mathrm{KCl}(\mathrm{aq})$ & $1.0892 \mathrm{E}-08$ & $1.0969 \mathrm{E}-08$ & -7.96 \\
\hline $\mathrm{Al}_{2}(\mathrm{OH})_{2} \mathrm{CO}_{3}^{+2}$ & $7.4845 \mathrm{E}-30$ & $3.9284 \mathrm{E}-30$ & -29.406 & $\mathrm{KH}_{2} \mathrm{PO}_{4}(\mathrm{aq})$ & $2.7566 \mathrm{E}-12$ & $2.7762 \mathrm{E}-12$ & -11.557 \\
\hline $\mathrm{Al}_{2} \mathrm{PO}_{4}^{+3}$ & $6.3258 \mathrm{E}-16$ & $1.4833 \mathrm{E}-16$ & -15.829 & $\mathrm{KHPO}_{4-}$ & $1.5684 \mathrm{E}-15$ & $1.3349 \mathrm{E}-15$ & -14.875 \\
\hline $\mathrm{Al}_{3}(\mathrm{OH})_{4}^{+5}$ & $7.8844 \mathrm{E}-22$ & $1.403 \mathrm{E}-23$ & -22.853 & $\mathrm{KNO}_{3}(\mathrm{aq})$ & $1.9491 \mathrm{E}-11$ & $1.963 \mathrm{E}-11$ & -10.707 \\
\hline $\mathrm{AlCl}^{+2}$ & $1.6503 \mathrm{E}-11$ & $8.662 \mathrm{E}-12$ & -11.062 & $\mathrm{KOH}(\mathrm{aq})$ & $1.1367 \mathrm{E}-15$ & $1.1447 \mathrm{E}-15$ & -14.941 \\
\hline $\mathrm{AlH}_{3} \mathrm{SiO}_{4}^{+2}$ & $1.5198 \mathrm{E}-10$ & $7.9769 \mathrm{E}-11$ & -10.098 & $\mathrm{KPO}_{4}^{-2}$ & $5.6987 \mathrm{E}-24$ & $2.9911 \mathrm{E}-24$ & -23.524 \\
\hline $\mathrm{AlHPO}_{4+}$ & $9.3566 \mathrm{E}-12$ & $7.964 \mathrm{E}-12$ & -11.099 & $\mathrm{KSO}_{4-}$ & 0.000001942 & 0.000001653 & -5.782 \\
\hline $\mathrm{AlOH}^{+2}$ & $1.5339 \mathrm{E}-09$ & $8.051 \mathrm{E}-10$ & -9.094 & $\mathrm{Mg}\left(\mathrm{NH}_{3}\right)_{2}{ }^{+2}$ & $2.6719 \mathrm{E}-23$ & $1.4024 \mathrm{E}-23$ & -22.853 \\
\hline $\mathrm{AlSO}_{4+}$ & $1.9368 \mathrm{E}-06$ & $1.6485 \mathrm{E}-06$ & -5.783 & $\mathrm{Mg}^{+2}$ & 0.00074463 & 0.00039083 & -3.408 \\
\hline $\mathrm{AsO}_{4}{ }^{-3}$ & $1.5138 \mathrm{E}-16$ & $3.5497 \mathrm{E}-17$ & -16.45 & $\mathrm{Mg}_{2} \mathrm{CO}_{3}^{+2}$ & $5.4696 \mathrm{E}-30$ & $2.8708 \mathrm{E}-30$ & -29.542 \\
\hline $\mathrm{Ca}\left(\mathrm{NH}_{3}\right)_{2}{ }^{+2}$ & $3.6995 \mathrm{E}-22$ & $1.9417 \mathrm{E}-22$ & -21.712 & $\mathrm{MgCl}+$ & $3.5997 \mathrm{E}-07$ & $3.064 \mathrm{E}-07$ & -6.514 \\
\hline $\mathrm{Ca}\left(\mathrm{NO}_{3}\right)_{2}$ & $3.2754 \mathrm{E}-21$ & $3.2987 \mathrm{E}-21$ & -20.482 & $\mathrm{MgCO}_{3}(\mathrm{aq})$ & $1.2674 \mathrm{E}-27$ & $1.2764 \mathrm{E}-27$ & -26.894 \\
\hline $\mathrm{Ca}^{+2}$ & 0.0030953 & 0.0016246 & -2.789 & $\mathrm{MgHCO}_{3+}$ & $3.1256 \mathrm{E}-22$ & $2.6604 \mathrm{E}-22$ & -21.575 \\
\hline $\mathrm{CaCl}+$ & $9.4413 \mathrm{E}-07$ & $8.036 \mathrm{E}-07$ & -6.095 & $\mathrm{MgHPO}_{4}(\mathrm{aq})$ & $4.7849 \mathrm{E}-13$ & $4.819 \mathrm{E}-13$ & -12.317 \\
\hline $\mathrm{CaCO}_{3}(\mathrm{aq})$ & $1.0784 \mathrm{E}-26$ & $1.086 \mathrm{E}-26$ & -25.964 & $\mathrm{MgOH}+$ & $1.0274 \mathrm{E}-12$ & $8.7446 \mathrm{E}-13$ & -12.058 \\
\hline $\mathrm{CaH}_{2} \mathrm{PO}_{4+}$ & $6.9924 \mathrm{E}-10$ & $5.9517 \mathrm{E}-10$ & -9.225 & $\mathrm{MgPO}_{4-}$ & $2.5075 \mathrm{E}-20$ & $2.1343 \mathrm{E}-20$ & -19.671 \\
\hline $\mathrm{CaHCO}_{3+}$ & $1.3162 \mathrm{E}-21$ & $1.1203 \mathrm{E}-21$ & -20.951 & $\mathrm{MgSO}_{4}(\mathrm{aq})$ & 0.00019802 & 0.00019943 & -3.7 \\
\hline $\mathrm{CaHPO}_{4}(\mathrm{aq})$ & $1.4409 \mathrm{E}-12$ & $1.4512 \mathrm{E}-12$ & -11.838 & $\mathrm{Mn}\left(\mathrm{NH}_{3}\right)_{2}^{+2}$ & $3.719 \mathrm{E}-22$ & $1.952 \mathrm{E}-22$ & -21.71 \\
\hline $\mathrm{CaNH}_{3}^{+2}$ & $1.9249 \mathrm{E}-12$ & $1.0103 \mathrm{E}-12$ & -11.996 & $\mathrm{Mn}\left(\mathrm{NH}_{3}\right)_{3}{ }^{+2}$ & $8.0717 \mathrm{E}-32$ & $4.2365 \mathrm{E}-32$ & -31.373 \\
\hline $\mathrm{CaNO}_{3+}$ & $1.6959 \mathrm{E}-09$ & $1.4435 \mathrm{E}-09$ & -8.841 & $\mathrm{Mn}\left(\mathrm{NH}_{3}\right)_{4}^{+2}$ & $9.1238 \mathrm{E}-42$ & $4.7888 \mathrm{E}-42$ & -41.32 \\
\hline $\mathrm{CaOH}+$ & $2.2442 \mathrm{E}-13$ & $1.9102 \mathrm{E}-13$ & -12.719 & $\mathrm{Mn}\left(\mathrm{NO}_{3}\right)_{2}(\mathrm{aq})$ & $1.1632 \mathrm{E}-16$ & $1.1715 \mathrm{E}-16$ & -15.931 \\
\hline $\mathrm{CaPO}_{4}^{-}$ & $6.6682 \mathrm{E}-18$ & $5.6757 \mathrm{E}-18$ & -17.246 & $\mathrm{Mn}(\mathrm{OH})_{4}^{-2}$ & $6.9077 \mathrm{E}-39$ & $3.6256 \mathrm{E}-39$ & -38.441 \\
\hline $\mathrm{CaSO}_{4}$ (aq) & 0.0010108 & 0.001018 & -2.992 & $\mathrm{Mn}^{+2}$ & 0.00084592 & 0.000444 & -3.353 \\
\hline $\mathrm{Cd}\left(\mathrm{CO}_{3}\right)_{2}^{-2}$ & $1.1596 \mathrm{E}-52$ & $6.0862 \mathrm{E}-53$ & -52.216 & $\mathrm{Mn}_{2}(\mathrm{OH})_{3+}$ & $2.3645 \mathrm{E}-21$ & $2.0126 \mathrm{E}-21$ & -20.696 \\
\hline $\mathrm{Cd}\left(\mathrm{NH}_{3}\right)_{2}{ }^{+2}$ & $3.6635 \mathrm{E}-22$ & $1.9229 \mathrm{E}-22$ & -21.716 & $\mathrm{Mn}_{2} \mathrm{OH}^{+3}$ & $4.2428 \mathrm{E}-14$ & $9.9485 \mathrm{E}-15$ & -14.002 \\
\hline $\mathrm{Cd}\left(\mathrm{NH}_{3}\right)_{3}{ }^{+2}$ & $1.4354 \mathrm{E}-30$ & $7.5338 \mathrm{E}-31$ & -30.123 & $\mathrm{MnCl}+$ & $1.1089 \mathrm{E}-07$ & $9.4386 \mathrm{E}-08$ & -7.025 \\
\hline $\mathrm{Cd}\left(\mathrm{NH}_{3}\right)_{4}^{+2}$ & $1.6565 \mathrm{E}-39$ & $8.6945 \mathrm{E}-40$ & -39.061 & $\mathrm{MnCl}_{2}(\mathrm{aq})$ & $3.5429 \mathrm{E}-11$ & $3.5681 \mathrm{E}-11$ & -10.448 \\
\hline $\mathrm{Cd}\left(\mathrm{NO}_{3}\right)_{2}(\mathrm{aq})$ & $1.5517 \mathrm{E}-20$ & $1.5627 \mathrm{E}-20$ & -19.806 & $\mathrm{MnCl}_{3-}$ & $2.4545 \mathrm{E}-15$ & $2.0892 \mathrm{E}-15$ & -14.68 \\
\hline $\mathrm{Cd}(\mathrm{OH})_{2}(\mathrm{aq})$ & $3.0846 \mathrm{E}-21$ & $3.1065 \mathrm{E}-21$ & -20.508 & $\mathrm{MnCO}_{3}(\mathrm{aq})$ & $1.0674 \mathrm{E}-25$ & $1.075 \mathrm{E}-25$ & -24.969 \\
\hline $\mathrm{Cd}(\mathrm{OH})_{3-}$ & $7.1824 \mathrm{E}-31$ & $6.1133 \mathrm{E}-31$ & -30.214 & $\mathrm{MnHCO}_{3+}$ & $6.5837 \mathrm{E}-22$ & $5.6038 \mathrm{E}-22$ & -21.252 \\
\hline $\mathrm{Cd}(\mathrm{OH})_{4}^{-2}$ & $2.3891 \mathrm{E}-41$ & $1.2539 \mathrm{E}-41$ & -40.902 & $\mathrm{MnHPO}_{4}(\mathrm{aq})$ & $2.1644 \mathrm{E}-12$ & $2.1798 \mathrm{E}-12$ & -11.662 \\
\hline $\mathrm{Cd}\left(\mathrm{SO}_{4}\right)_{2}{ }^{-2}$ & $9.0828 \mathrm{E}-09$ & $4.7672 \mathrm{E}-09$ & -8.322 & $\mathrm{MnNH}_{3}^{+2}$ & $9.3442 \mathrm{E}-13$ & $4.9044 \mathrm{E}-13$ & -12.309 \\
\hline $\mathrm{Cd}^{+2}$ & $2.9257 \mathrm{E}-07$ & $1.5356 \mathrm{E}-07$ & -6.814 & $\mathrm{MnNO}_{3+}$ & $2.0949 \mathrm{E}-10$ & $1.7831 \mathrm{E}-10$ & -9.749 \\
\hline $\mathrm{Cd}_{2} \mathrm{OH}^{+3}$ & $3.3483 \mathrm{E}-20$ & $7.8512 \mathrm{E}-21$ & -20.105 & $\mathrm{MnOH}+$ & $8.3835 \mathrm{E}-12$ & $7.1357 \mathrm{E}-12$ & -11.147 \\
\hline $\mathrm{CdCl}+$ & $3.5252 \mathrm{E}-09$ & $3.0005 \mathrm{E}-09$ & -8.523 & $\mathrm{MnSO}_{4}(\mathrm{aq})$ & 0.00020797 & 0.00020945 & -3.679 \\
\hline $\mathrm{CdCl}_{2}(\mathrm{aq})$ & $2.5121 \mathrm{E}-12$ & $2.53 \mathrm{E}-12$ & -11.597 & $\mathrm{Na}^{+1}$ & 0.00056343 & 0.00047957 & -3.319 \\
\hline $\mathrm{CdCO}_{3}(\mathrm{aq})$ & $1.7268 \mathrm{E}-29$ & $1.7391 \mathrm{E}-29$ & -28.76 & $\mathrm{Na}_{2} \mathrm{HPO}_{4}(\mathrm{aq})$ & $3.7125 \mathrm{E}-18$ & $3.7388 \mathrm{E}-18$ & -17.427 \\
\hline $\mathrm{CdHCO}_{3+}$ & $2.9533 \mathrm{E}-25$ & $2.5137 \mathrm{E}-25$ & -24.6 & $\mathrm{Na}_{2} \mathrm{PO}_{4-}$ & $1.632 \mathrm{E}-25$ & $1.3891 \mathrm{E}-25$ & -24.857 \\
\hline $\mathrm{CdHPO}_{4}(\mathrm{aq})$ & $1.4264 \mathrm{E}-15$ & $1.4365 \mathrm{E}-15$ & -14.843 & $\mathrm{NaCl}(\mathrm{aq})$ & $5.9125 \mathrm{E}-08$ & $5.9545 \mathrm{E}-08$ & -7.225 \\
\hline
\end{tabular}


Table 6 (continued)

\begin{tabular}{|c|c|c|c|c|c|c|c|}
\hline Species & Concentration & Activity & Log activity & Species & Concentration & Activity & Log activity \\
\hline $\mathrm{CdNH}_{3}{ }^{+2}$ & $1.895 \mathrm{E}-14$ & $9.946 \mathrm{E}-15$ & -14.002 & $\mathrm{NaCO}_{3-}$ & $7.4809 \mathrm{E}-29$ & $6.3674 \mathrm{E}-29$ & -28.196 \\
\hline $\mathrm{CdNO}_{3+}$ & $2.1605 \mathrm{E}-13$ & $1.8389 \mathrm{E}-13$ & -12.735 & $\mathrm{NaH}_{2} \mathrm{PO}_{4}(\mathrm{aq})$ & $1.3862 \mathrm{E}-11$ & $1.396 \mathrm{E}-11$ & -10.855 \\
\hline $\mathrm{CdOH}+$ & $1.0089 \mathrm{E}-14$ & $8.5877 \mathrm{E}-15$ & -14.066 & $\mathrm{NaHCO}_{3}(\mathrm{aq})$ & $2.1193 \mathrm{E}-23$ & $2.1344 \mathrm{E}-23$ & -22.671 \\
\hline $\mathrm{CdSO}_{4}(\mathrm{aq})$ & $9.4822 \mathrm{E}-08$ & $9.5496 \mathrm{E}-08$ & -7.02 & $\mathrm{NaHPO}_{4-}$ & $1.2215 \mathrm{E}-14$ & $1.0397 \mathrm{E}-14$ & -13.983 \\
\hline $\mathrm{Cl}^{-1}$ & 0.00024976 & 0.00021258 & -3.672 & $\mathrm{NaNO}_{3}(\mathrm{aq})$ & $3.4007 \mathrm{E}-11$ & $3.4249 \mathrm{E}-11$ & -10.465 \\
\hline $\mathrm{CO}_{3}^{-2}$ & $9.2043 \mathrm{E}-27$ & $4.831 \mathrm{E}-27$ & -26.316 & $\mathrm{NaOH}(\mathrm{aq})$ & $3.8356 \mathrm{E}-15$ & $3.8628 \mathrm{E}-15$ & -14.413 \\
\hline $\mathrm{Cu}\left(\mathrm{CO}_{3}\right)_{2}{ }^{-2}$ & $5.6164 \mathrm{E}-51$ & $2.9478 \mathrm{E}-51$ & -50.53 & $\mathrm{NaPO}_{4}^{-2}$ & $3.2762 \mathrm{E}-23$ & $1.7196 \mathrm{E}-23$ & -22.765 \\
\hline $\mathrm{Cu}\left(\mathrm{NH}_{3}\right)_{2}{ }^{+2}$ & $1.7593 \mathrm{E}-20$ & $9.2342 \mathrm{E}-21$ & -20.035 & $\mathrm{NaSO}_{4-}$ & $9.5175 \mathrm{E}-06$ & $8.1009 \mathrm{E}-06$ & -5.091 \\
\hline $\mathrm{Cu}\left(\mathrm{NH}_{3}\right)_{3}{ }^{+2}$ & $2.366 \mathrm{E}-27$ & $1.2419 \mathrm{E}-27$ & -26.906 & $\mathrm{NH}_{3}(\mathrm{aq})$ & $1.4195 \mathrm{E}-10$ & $1.4296 \mathrm{E}-10$ & -9.845 \\
\hline $\mathrm{Cu}\left(\mathrm{NH}_{3}\right)_{4}^{+2}$ & $6.7235 \mathrm{E}-35$ & $3.5289 \mathrm{E}-35$ & -34.452 & $\mathrm{NH}_{4}^{+1}$ & 0.00040152 & 0.00034176 & -3.466 \\
\hline $\mathrm{Cu}\left(\mathrm{NO}_{3}\right)_{2}(\mathrm{aq})$ & $2.0228 \mathrm{E}-22$ & $2.0372 \mathrm{E}-22$ & -21.691 & $\mathrm{NH}_{4} \mathrm{SO}_{4-}$ & 0.00001348 & 0.000011474 & -4.94 \\
\hline $\mathrm{Cu}(\mathrm{OH})_{2}(\mathrm{aq})$ & $3.1248 \mathrm{E}-19$ & $3.147 \mathrm{E}-19$ & -18.502 & $\mathrm{NO}_{3}^{-1}$ & $2.9771 \mathrm{E}-07$ & $2.5339 \mathrm{E}-07$ & -6.596 \\
\hline $\mathrm{Cu}(\mathrm{OH})_{3}-$ & $1.7038 \mathrm{E}-25$ & $1.4502 \mathrm{E}-25$ & -24.839 & $\mathrm{OH}-$ & $8.1149 \mathrm{E}-12$ & $6.9071 \mathrm{E}-12$ & -11.161 \\
\hline $\mathrm{Cu}(\mathrm{OH})_{4}^{-2}$ & $1.4733 \mathrm{E}-36$ & $7.7328 \mathrm{E}-37$ & -36.112 & $\mathrm{~Pb}\left(\mathrm{CO}_{3}\right)_{2}^{-2}$ & $2.7727 \mathrm{E}-50$ & $1.4553 \mathrm{E}-50$ & -49.837 \\
\hline $\mathrm{Cu}^{+2}$ & $1.5184 \mathrm{E}-08$ & $7.9696 \mathrm{E}-09$ & -8.099 & $\mathrm{~Pb}\left(\mathrm{NO}_{3}\right)_{2}(\mathrm{aq})$ & $1.3009 \mathrm{E}-19$ & $1.3101 \mathrm{E}-19$ & -18.883 \\
\hline $\mathrm{Cu}_{2}(\mathrm{OH})_{2}^{+2}$ & $3.7945 \mathrm{E}-21$ & $1.9916 \mathrm{E}-21$ & -20.701 & $\mathrm{~Pb}(\mathrm{OH})_{2}(\mathrm{aq})$ & $2.2793 \mathrm{E}-18$ & $2.2955 \mathrm{E}-18$ & -17.639 \\
\hline $\mathrm{Cu}_{2} \mathrm{OH}^{+3}$ & $6.2868 \mathrm{E}-20$ & $1.4741 \mathrm{E}-20$ & -19.831 & $\mathrm{~Pb}(\mathrm{OH})_{3-}$ & $5.4183 \mathrm{E}-26$ & $4.6119 \mathrm{E}-26$ & -25.336 \\
\hline $\mathrm{Cu}_{3}(\mathrm{OH})_{4}^{+2}$ & $3.2625 \mathrm{E}-33$ & $1.7123 \mathrm{E}-33$ & -32.766 & $\mathrm{~Pb}\left(\mathrm{SO}_{4}\right)_{2}{ }^{-2}$ & $3.952 \mathrm{E}-09$ & $2.0743 \mathrm{E}-09$ & -8.683 \\
\hline $\mathrm{CuCl}+$ & $3.3884 \mathrm{E}-12$ & $2.8841 \mathrm{E}-12$ & -11.54 & $\mathrm{~Pb}^{+2}$ & $1.364 \mathrm{E}-07$ & $7.1594 \mathrm{E}-08$ & -7.145 \\
\hline $\mathrm{CuCl}_{2}$ (aq) & $8.4395 \mathrm{E}-17$ & $8.4995 \mathrm{E}-17$ & -16.071 & $\mathrm{~Pb}_{2} \mathrm{OH}^{+3}$ & $1.7484 \mathrm{E}-17$ & $4.0997 \mathrm{E}-18$ & -17.387 \\
\hline $\mathrm{CuCl}_{3-}$ & $1.5421 \mathrm{E}-22$ & $1.3125 \mathrm{E}-22$ & -21.882 & $\mathrm{~Pb}_{3}(\mathrm{OH})_{4}^{+2}$ & $1.5516 \mathrm{E}-33$ & $8.1438 \mathrm{E}-34$ & -33.089 \\
\hline $\mathrm{CuCl}_{4}{ }_{1}^{-2}$ & $4.2762 \mathrm{E}-28$ & $2.2444 \mathrm{E}-28$ & -27.649 & $\mathrm{~Pb}_{4}(\mathrm{OH})_{4}^{+4}$ & $1.3902 \mathrm{E}-36$ & $1.055 \mathrm{E}-37$ & -36.977 \\
\hline $\mathrm{CuCO}_{3}(\mathrm{aq})$ & $2.2511 \mathrm{E}-28$ & $2.2671 \mathrm{E}-28$ & -27.645 & $\mathrm{PbCl}+$ & $5.4968 \mathrm{E}-10$ & $4.6787 \mathrm{E}-10$ & -9.33 \\
\hline $\mathrm{CuHCO}_{3+}$ & $3.0511 \mathrm{E}-26$ & $2.597 \mathrm{E}-26$ & -25.586 & $\mathrm{PbCl}_{2}$ (aq) & $2.0284 \mathrm{E}-13$ & $2.0428 \mathrm{E}-13$ & -12.69 \\
\hline $\mathrm{CuHPO}_{4}$ (aq) & $1.9472 \mathrm{E}-16$ & $1.961 \mathrm{E}-16$ & -15.708 & $\mathrm{PbCl}_{3-}$ & $4.0528 \mathrm{E}-17$ & $3.4496 \mathrm{E}-17$ & -16.462 \\
\hline $\mathrm{CuHSO}_{4+}$ & $3.2167 \mathrm{E}-12$ & $2.738 \mathrm{E}-12$ & -11.563 & $\mathrm{PbCl}_{4}^{-2}$ & $5.0348 \mathrm{E}-21$ & $2.6426 \mathrm{E}-21$ & -20.578 \\
\hline $\mathrm{CuNH}_{3}{ }^{+2}$ & $3.3824 \mathrm{E}-14$ & $1.7753 \mathrm{E}-14$ & -13.751 & $\mathrm{PbCO}_{3}(\mathrm{aq})$ & $1.1637 \mathrm{E}-27$ & $1.172 \mathrm{E}-27$ & -26.931 \\
\hline $\mathrm{CuNO}_{3+}$ & $8.115 \mathrm{E}-15$ & $6.9072 \mathrm{E}-15$ & -14.161 & $\mathrm{PbH}_{2} \mathrm{PO}_{4+}$ & $3.8807 \mathrm{E}-14$ & $3.3031 \mathrm{E}-14$ & -13.481 \\
\hline $\mathrm{CuOH}+$ & $2.9984 \mathrm{E}-13$ & $2.5521 \mathrm{E}-13$ & -12.593 & $\mathrm{PbHCO}_{3+}$ & $3.4586 \mathrm{E}-24$ & $2.9438 \mathrm{E}-24$ & -23.531 \\
\hline $\mathrm{CuSO}_{4}(\mathrm{aq})$ & $4.8091 \mathrm{E}-09$ & $4.8433 \mathrm{E}-09$ & -8.315 & $\mathrm{PbHPO}_{4}(\mathrm{aq})$ & $1.6514 \mathrm{E}-16$ & $1.6631 \mathrm{E}-16$ & -15.779 \\
\hline $\mathrm{Fe}\left(\mathrm{NH}_{3}\right)_{2}{ }^{+2}$ & $1.8136 \mathrm{E}-21$ & $9.5187 \mathrm{E}-22$ & -21.021 & $\mathrm{PbNO}_{3+}$ & $3.2755 \mathrm{E}-13$ & $2.788 \mathrm{E}-13$ & -12.555 \\
\hline $\mathrm{Fe}\left(\mathrm{NH}_{3}\right)_{3}{ }^{+2}$ & $8.2541 \mathrm{E}-31$ & $4.3323 \mathrm{E}-31$ & -30.363 & $\mathrm{PbOH}+$ & $2.6917 \mathrm{E}-12$ & $2.2911 \mathrm{E}-12$ & -11.64 \\
\hline $\mathrm{Fe}\left(\mathrm{NH}_{3}\right)_{4}^{+2}$ & $1.6025 \mathrm{E}-40$ & $8.4111 \mathrm{E}-41$ & -40.075 & $\mathrm{PbSO}_{4}(\mathrm{aq})$ & $1.0909 \mathrm{E}-07$ & $1.0987 \mathrm{E}-07$ & -6.959 \\
\hline $\mathrm{Fe}(\mathrm{OH})_{2}(\mathrm{aq})$ & $2.5099 \mathrm{E}-19$ & $2.5277 \mathrm{E}-19$ & -18.597 & $\mathrm{PO}_{4}^{-3}$ & $6.6212 \mathrm{E}-21$ & $1.5525 \mathrm{E}-21$ & -20.809 \\
\hline $\mathrm{Fe}(\mathrm{OH})_{2+}$ & 0.00023557 & 0.00020051 & -3.698 & $\mathrm{SO}_{4}^{-2}$ & 0.0059696 & 0.0031332 & -2.504 \\
\hline $\mathrm{Fe}(\mathrm{OH})_{3-}$ & $1.6563 \mathrm{E}-26$ & $1.4098 \mathrm{E}-26$ & -25.851 & $\mathrm{Sr}^{+2}$ & $8.7518 \mathrm{E}-06$ & $4.5935 \mathrm{E}-06$ & -5.338 \\
\hline $\mathrm{Fe}(\mathrm{OH})_{3}(\mathrm{aq})$ & $1.0881 \mathrm{E}-10$ & $1.0958 \mathrm{E}-10$ & -9.96 & $\mathrm{SrCl}+$ & $1.5247 \mathrm{E}-09$ & $1.2978 \mathrm{E}-09$ & -8.887 \\
\hline $\mathrm{Fe}(\mathrm{OH})_{4-}$ & $1.1199 \mathrm{E}-15$ & $9.532 \mathrm{E}-16$ & -15.021 & $\mathrm{SrCO}_{3}(\mathrm{aq})$ & $9.5196 \mathrm{E}-30$ & $9.5873 \mathrm{E}-30$ & -29.018 \\
\hline $\mathrm{Fe}\left(\mathrm{SO}_{4}\right)_{2-}$ & 0.00011164 & 0.000095023 & -4.022 & $\mathrm{SrH}_{2} \mathrm{PO}_{4+}$ & $5.2866 \mathrm{E}-13$ & $4.4997 \mathrm{E}-13$ & -12.347 \\
\hline $\mathrm{Fe}^{+2}$ & 0.00037202 & 0.00019526 & -3.709 & $\mathrm{SrHCO}_{3+}$ & $3.7047 \mathrm{E}-24$ & $3.1533 \mathrm{E}-24$ & -23.501 \\
\hline $\mathrm{Fe}^{+3}$ & 0.00024847 & 0.00005826 & -4.235 & $\mathrm{SrHPO}_{4}(\mathrm{aq})$ & $1.9503 \mathrm{E}-15$ & $1.9642 \mathrm{E}-15$ & -14.707 \\
\hline $\mathrm{Fe}_{2}(\mathrm{OH})_{2}{ }^{+4}$ & 0.000077229 & $5.8609 \mathrm{E}-06$ & -5.232 & $\mathrm{SrNH}_{3}^{+2}$ & $3.4341 \mathrm{E}-15$ & $1.8025 \mathrm{E}-15$ & -14.744 \\
\hline $\mathrm{Fe}_{3}(\mathrm{OH})_{4}^{+5}$ & 0.000026047 & $4.635 \mathrm{E}-07$ & -6.334 & $\mathrm{SrNO}_{3+}$ & $6.592 \mathrm{E}-12$ & $5.6109 \mathrm{E}-12$ & -11.251 \\
\hline $\mathrm{FeCl}+$ & $3.077 \mathrm{E}-08$ & $2.619 \mathrm{E}-08$ & -7.582 & $\mathrm{SrOH}+$ & $2.2381 \mathrm{E}-16$ & $1.905 \mathrm{E}-16$ & -15.72 \\
\hline
\end{tabular}


Table 6 (continued)

\begin{tabular}{|c|c|c|c|c|c|c|c|}
\hline Species & Concentration & Activity & Log activity & Species & Concentration & Activity & Log activity \\
\hline $\mathrm{FeCl}^{+2}$ & $4.5894 \mathrm{E}-07$ & $2.4088 \mathrm{E}-07$ & -6.618 & $\mathrm{SrSO}_{4}(\mathrm{aq})$ & $2.4468 \mathrm{E}-06$ & $2.4642 \mathrm{E}-06$ & -5.608 \\
\hline $\mathrm{FeH}_{2} \mathrm{PO}_{4}+$ & $1.6774 \mathrm{E}-09$ & $1.4277 \mathrm{E}-09$ & -8.845 & $\mathrm{Zn}\left(\mathrm{CO}_{3}\right)_{2}^{-2}$ & $3.5822 \mathrm{E}-49$ & $1.8802 \mathrm{E}-49$ & -48.726 \\
\hline $\mathrm{FeH}_{2} \mathrm{PO}_{4}^{+2}$ & $3.0645 \mathrm{E}-08$ & $1.6085 \mathrm{E}-08$ & -7.794 & $\mathrm{Zn}\left(\mathrm{NH}_{3}\right)_{2}^{+2}$ & $7.7717 \mathrm{E}-19$ & $4.0791 \mathrm{E}-19$ & -18.389 \\
\hline $\mathrm{FeHCO}_{3+}$ & $1.4915 \mathrm{E}-22$ & $1.2695 \mathrm{E}-22$ & -21.896 & $\mathrm{Zn}\left(\mathrm{NH}_{3}\right)_{3}{ }^{+2}$ & $3.5763 \mathrm{E}-26$ & $1.8771 \mathrm{E}-26$ & -25.727 \\
\hline $\mathrm{FeHPO}_{4}(\mathrm{aq})$ & $1.4242 \mathrm{E}-12$ & $1.4343 \mathrm{E}-12$ & -11.843 & $\mathrm{Zn}\left(\mathrm{NH}_{3}\right)_{4}^{+2}$ & $8.2616 \mathrm{E}-34$ & $4.3362 \mathrm{E}-34$ & -33.363 \\
\hline $\mathrm{FeHPO}_{4+}$ & $1.8415 \mathrm{E}-06$ & $1.5674 \mathrm{E}-06$ & -5.805 & $\mathrm{Zn}\left(\mathrm{NO}_{3}\right)_{2}(\mathrm{aq})$ & $1.2902 \mathrm{E}-17$ & $1.2993 \mathrm{E}-17$ & -16.886 \\
\hline $\mathrm{FeNH}_{3}{ }^{+2}$ & $1.5771 \mathrm{E}-12$ & $8.2778 \mathrm{E}-13$ & -12.082 & $\mathrm{Zn}(\mathrm{OH})_{2}(\mathrm{aq})$ & $2.0373 \mathrm{E}-14$ & $2.0518 \mathrm{E}-14$ & -13.688 \\
\hline $\mathrm{FeOH}+$ & $6.308 \mathrm{E}-11$ & $5.3691 \mathrm{E}-11$ & -10.27 & $\mathrm{Zn}(\mathrm{OH})_{3-}$ & $1.5315 \mathrm{E}-22$ & $1.3036 \mathrm{E}-22$ & -21.885 \\
\hline $\mathrm{FeOH}^{+2}$ & 0.0013085 & 0.00068679 & -3.163 & $\mathrm{Zn}(\mathrm{OH})_{4}^{-2}$ & $7.9083 \mathrm{E}-32$ & $4.1508 \mathrm{E}-32$ & -31.382 \\
\hline $\mathrm{FeSO}_{4}(\mathrm{aq})$ & 0.00012795 & 0.00012886 & -3.89 & $\mathrm{Zn}\left(\mathrm{SO}_{4}\right)_{2}{ }^{-2}$ & 0.00001439 & $7.5529 \mathrm{E}-06$ & -5.122 \\
\hline $\mathrm{FeSO}_{4+}$ & 0.0023639 & 0.0020121 & -2.696 & $\mathrm{Zn}^{+2}$ & 0.00076928 & 0.00040377 & -3.394 \\
\hline $\mathrm{H}^{+1}$ & 0.00058883 & 0.00050119 & -3.3 & $\mathrm{Zn}_{2} \mathrm{OH}^{+3}$ & $4.1208 \mathrm{E}-13$ & $9.6623 \mathrm{E}-14$ & -13.015 \\
\hline $\mathrm{H}_{2} \mathrm{AsO}_{4-}$ & 0.000096936 & 0.000082508 & -4.084 & $\mathrm{ZnCl+}$ & $2.6229 \mathrm{E}-07$ & $2.2325 \mathrm{E}-07$ & -6.651 \\
\hline $\mathrm{H}_{2} \mathrm{CO}_{3} *(\mathrm{aq})$ & $9.9913 \mathrm{E}-17$ & $1.0062 \mathrm{E}-16$ & -15.997 & $\mathrm{ZnCl}_{2}$ (aq) & $2.5842 \mathrm{E}-11$ & $2.6026 \mathrm{E}-11$ & -10.585 \\
\hline $\mathrm{H}_{2} \mathrm{PO}_{4-}$ & $2.4187 \mathrm{E}-08$ & $2.0587 \mathrm{E}-08$ & -7.686 & $\mathrm{ZnCl}_{3-}$ & $6.7044 \mathrm{E}-15$ & $5.7066 \mathrm{E}-15$ & -14.244 \\
\hline $\mathrm{H}_{2} \mathrm{SiO}_{4}^{-2}$ & $1.9947 \mathrm{E}-21$ & $1.047 \mathrm{E}-21$ & -20.98 & $\mathrm{ZnCl}_{4}{ }^{-2}$ & $1.0348 \mathrm{E}-18$ & $5.4311 \mathrm{E}-19$ & -18.265 \\
\hline $\mathrm{H}_{3} \mathrm{AsO}_{4}$ & 0.000007034 & 0.000007084 & -5.15 & $\mathrm{ZnCO}_{3}(\mathrm{aq})$ & $1.1145 \mathrm{E}-25$ & $1.1224 \mathrm{E}-25$ & -24.95 \\
\hline $\mathrm{H}_{3} \mathrm{PO}_{4}$ & $1.248 \mathrm{E}-09$ & $1.2569 \mathrm{E}-09$ & -8.901 & $\mathrm{ZnHCO}_{3+}$ & $7.7473 \mathrm{E}-22$ & $6.5942 \mathrm{E}-22$ & -21.181 \\
\hline $\mathrm{H}_{3} \mathrm{SiO}_{4-}$ & $5.5466 \mathrm{E}-11$ & $4.721 \mathrm{E}-11$ & -10.326 & $\mathrm{ZnHPO}_{4}(\mathrm{aq})$ & $1.5279 \mathrm{E}-12$ & $1.5388 \mathrm{E}-12$ & -11.813 \\
\hline $\mathrm{H}_{4} \mathrm{SiO}_{4}$ & 0.00034043 & 0.00034285 & -3.465 & $\mathrm{ZnNH}_{3}{ }^{+2}$ & $2.192 \mathrm{E}-11$ & $1.1505 \mathrm{E}-11$ & -10.939 \\
\hline $\mathrm{H}_{4} \mathrm{SiO}_{4} \mathrm{SO}_{4}^{-2}$ & $5.6811 \mathrm{E}-07$ & $2.9818 \mathrm{E}-07$ & -6.526 & $\mathrm{ZnNO}_{3+}$ & $3.2971 \mathrm{E}-10$ & $2.8064 \mathrm{E}-10$ & -9.552 \\
\hline $\mathrm{HAsO}_{4}^{-2}$ & $3.0294 \mathrm{E}-08$ & $1.59 \mathrm{E}-08$ & -7.799 & $\mathrm{ZnOH}+$ & $3.2765 \mathrm{E}-10$ & $2.7888 \mathrm{E}-10$ & -9.555 \\
\hline $\mathrm{HCO}_{3-}$ & $8.3841 \mathrm{E}-20$ & $7.1362 \mathrm{E}-20$ & -19.147 & $\mathrm{ZnSO}_{4}(\mathrm{aq})$ & 0.00024408 & 0.00024581 & -3.609 \\
\hline
\end{tabular}

Heavy metals such as $\mathrm{Fe}$ and also $\mathrm{Al}$, and metalloids such as As, are known to be toxic to plants. Fe toxicity is mainly a problem under reduced conditions, because the oxidized form $\mathrm{Fe}(\mathrm{III})$ is much less soluble than the reduced $\mathrm{Fe}(\mathrm{II})$ form. In the terrace walls, $\mathrm{Fe}$ is relatively harmless due to its precipitation as schwertmannite-type material. Al is always toxic if present in large quantities, but concentrations in the stream water are low. Moreover, the precipitation of $\mathrm{Fe}$ and $\mathrm{Al}$ hydroxides in the early phase described above and prominent ion pair formation (data not shown) must lead to very reduced concentrations of dissolved $\mathrm{Al}^{3+}$ and $\mathrm{Fe}^{3+}$ near the living moss (see, e.g. España 2007). Arsenic may also be very toxic, partly because it is disrupting Pdependent aspects of metabolism (Finnegan and Chen 2012), but toxic levels in the vicinity of the living moss parts are most probably inhibited by the sorption of As to the schwertmannite-type precipitate.

\section{Conclusions}

In the harsh aquatic environment of the Rio Santiago streambed, on living outer parts of the single higher plant species, the rare moss $A$. prostratum (Müll. Hal.) Besch, a coating of $\mathrm{Al}$ and $\mathrm{Fe}$ hydroxides is formed, which can be attributed to local supersaturation as a result of prevalent adsorption of trivalent cations by the living moss. Continued deposition ultimately induces its senescence and death, whereas newly formed leaves temporarily escape such fate. On the necromass and in the stream water, schwertmannite-type material precipitates to form moss necromass coatings and sediment particles that also contain 'captured' fine detrital primary minerals. Sorption or fixation of As by this material is prominent, leads to levels of up to $3.5 \%$ As and most probably limits the concentration of dissolved arsenate. This is assumed to create a local, far less toxic environment around the living moss that allows this particular species-A. prostratum - to survive. 
The sequence of processes explains the peculiar terraceforming growth of the moss, i.e. analogue to travertineterrace formation in highly calcareous streams. Remarkably, this case study seems to be the first more detailed study on moss-built microterraces in a truly acid, aquatic environment.

Bryophytes have no roots and thus escape the encrusted and toxic parts of the terrace walls. Some species are more tolerant to heavy metals and As than others, especially calcifuge species (Bates 1978) or have developed particular tolerances to heavy metals (Shaw 198 7). Anyway, heavy metal concentrations in our moss are low and do not exhibit a clear relation with their concentrations in the stream water. Moreover, our study shows that estimates of these plant concentrations are problematic due to the presence of coatings and crusts and that results strongly depend on the pretreatment of the plant material. This calls for a critical evaluation of the bioindicator value of bryophytes in such polluted aquatic environment (see e.g. Samecka-Cymerman et al. 2002).

Solute concentrations and precipitation in the highly acid stream water are typical for acid mine drainage-polluted rivers (high in ferric iron, sulphate and calcium; schwertmannite-type precipitate) and have been so for a prolonged time, evidenced by the rather invariable composition of interstitial sediment from a moss-built terrace rim. Results furthermore show that the solute concentration of As is a poor indicator for the stream water quality, since it lies far below the levels encountered in fine sediment, which abounds in this water (as suspended material) and in the river deposits. Evidently, it would be hazardous to judge environmental risks of the use of such stream water by its solute composition and to overlook this fine, suspended load.

Acknowledgments Thanks are due to Dr. William R. Buck from the New York Botanical Garden for the identification of the moss and to Dr. Eberhard Hegewald for his help with retrieving the original papers on the moss species of Ancash, including the occurrence of the A. prostratum we now found in the Rio Santiago. We were also aided with its identification by Drs. Ing. Guido van Reenen and Prof. Dr. Toine (A.M.) Cleef from IBED, University of Amsterdam. X-ray diffraction and X-ray fluorescence spectroscopy (XRF) analyses were performed by the Geolab of the Faculty of Geosciences, University of Utrecht, the Netherlands. Leen de Lange and Leo Hoitinga, from IBED, are acknowledged for part of the chemical analyses. We further acknowledge Jan van Arkel
(IBED) for his technical assistance in the production of microscopic photographs of the moss and the other illustrations.

Conflict of Interest The authors declare that they have no conflict of interest.

Open Access This article is distributed under the terms of the Creative Commons Attribution 4.0 International License (http:// creativecommons.org/licenses/by/4.0/), which permits unrestricted use, distribution, and reproduction in any medium, provided you give appropriate credit to the original author(s) and the source, provide a link to the Creative Commons license, and indicate if changes were made.

\section{References}

Bates, J. W. (1978). The influence of metal availability on the bryophyte and macro-lichen vegetation of four rock types on Skye and Rhum. Journal of Ecology, 66, 457-82.

Bigham, J. M., Schwertmann, U., Carlson, L., \& Murad, E. (1990). A poorly crystallized oxyhydroxysulfate of iron formed by bacterial oxidation of Fe(II) in acid mine waters. Geochimica et Cosmochimica Acta, 54, 2743-2758.

Bigham, J. M., Schwertmann, U., Trainable, S. J., Winland, R. L., \& Wolf, M. (1996). Schwertmannite and the chemical modeling of iron in acid sulfate waters. Geochimica et Cosmochimica Acta, 60, 2111-2121.

Bodenlos, A.J., Straczek, J.A. (1957). Base metal deposits of the Cordillera Negra, Departamento de Ancash, Peru. US Geological Survey Bulletin, 1040, 165p.

Carlson, L., Bigham, J. M., Schwertmann, U., Kyek, A., \& Wagner, F. (2002). Scavenging of As from acid mine drainage by schwertmannite and ferrihydrite: a comparison with synthetic analogues. Environmental Science \& Technology, 36, 1712-1719.

Chirif, H., Carlotto, V., Fidel, L. (2010). Generalidades de la evaluación del potencial minero regional. 2010. http://zeeot. regioncajamarca.gob.pe/rrnn-no-renovables. Accessed 18 October 2014.

Erten-Unal, M., Wixson, B. G., Gale, N., \& Pitt, J. L. (1998). Evaluation of toxicity, bioavailability and speciation of lead, zinc and cadmium in mine/mill wastewaters. Chemical Speciation \& Bioavailability, 10, 37-46.

España, J. S. (2007). The behavior of iron and aluminum in acid mine drainage: speciation, mineralogy, and environmental significance. In T. M. Letcher (Ed.), Thermodynamics, solubility and environmental issues (pp. 137-150). Amsterdam: Elsevier.

España, J. S., Pamo, E. L., Santofimia, E., Aduvire, O., Reyes, J., \& Barettino, D. (2005). Acid mine drainage in the Iberian Pyrite Belt (Odiel river watershed, Huelva, SW Spain). Applied Geochemistry, 20, 1320-1356.

España, J. S., Pastor, E. S., \& Pamo, E. L. (2007). Iron terraces in acid mine drainage systems: a discussion about the organic and inorganic factors involved in their formation through 
observations from the Tintillo acidic river (Riotinto mine, Huelva, Spain). Geosphere, 3, 133-151.

Finnegan, P.M., Chen, W. (2012). Arsenic toxicity: the effects on plant metabolism. Frontiers in physiology, 3 (182), 1-14. http://www.ncbi.nlm.nih.gov/pmc/articles/PMC3368394/ pdf/fphys-03-00182.pdf.

Fukushi, K., Sato, T., \& Yanase, N. (2003). Solid-solution reactions in $\mathrm{As}(\mathrm{V})$ sorption by schwertmannite. Environmental Science \& Technology, 37, 3581-3586.

GBIF. Biodiversity occurrence data of Anomobryum prostratum published by: the New York Botanical Garden, Comision nacional para el conocimiento y uso de la biodiversidad, Instituto de Ciencias Naturalis, Missouri Botanical Garden and Administracion de Parques Nacionales Argentina 2013. Accessed through GBIF Data Portal, data.gbif.org, 21 May 2013.

Guo, C. Q., Ochyra, R., Wu, P. C., Seppelt, R. D., Yao, Y. F., Bian, L. G., Li, S. P., \& Li, C. S. (2013). Warnstorfia exannulata, an aquatic moss in the Arctic: seasonal growth responses. Climatic Change, 119, 407-419.

Hegewald, E., \& Hegewald, P. (1977). Eine Moossammlung aus Peru III. Nova Hedwigia, 287, 31-758.

Hegewald, E., \& Hegewald, P. (1985). Eine Moossammlung aus Peru I. Nova Hedwigia, 41, 219-271.

Ilyashuk, B. P. (2002). Growth and production of aquatic mosses in acidified lakes of Karelia Republic, Russia. Water, Air, and Soil Pollution, 135, 285-290.

Jackson, M. L. (1956). Soil chemical analysis-advanced course. Madison: Jackson.

Jacobsen, D. (2011). Tropical high-altitude streams. In D. Dudgeon (Ed.), Tropical stream ecology (pp. 219-256). San Diego: Academic.

Lang, J., Lucas, G. (1970). Contribution à l'étude de biohermes continentaux: barrages des lacs de Band-e-Amir (Afghanistan central). Bulletin de la Societe geologique de France, 7 (XII-5), 834-842.

Loayza-Muro, R. A., Elias-Letts, R., Marticorena-Ruiz, J. K., Palomino, E. J., Duivenvoorden, J. F., Kraak, H. S., \& Admiraal, W. (2010). Metal-induced shifts in benthic macro invertebrate community composition in Andean high altitude streams. Environmental Toxicology and Chemistry, 29, 2761-2768.

Monsalve, C., \& Cano, A. (2003). La familia Brassicaceae en la provincia de Huaylas, Ancash. Revista Peruana de Biología, $10,20-32$.

Morin, G., Juillot, F., Casiot, C., Bruneel, O., Personné, J. C., Elbaz-Poulichet, F., Leblanc, M., Ildefonse, P., \& Calas, G. (2003). Bacterial formation of tooeleite and mixed arsenic(III) or arsenic(V)-iron(III) gels in the Carnoulès acid mine drainage, France. A XANES, XRD, and SEM study. Environmental Science \& Technology, 37, 1705-12.

MRC1 (n.d). MRC1 Exploraciones, Prospecto AIJA. Not dated. http://www.emrcexploraciones.com/pdf/AIJA.pdf.

Murad, E., Schwertmann, U., Bigham, J.M., Carlson, L. (1994). Mineralogical characteristics of poorly crystallized precipitates formed by oxidation of $\mathrm{Fe}^{2+}$ in acid sulfate waters. In: C.N. Alpers, D. Blowes (Ed), The environmental geochemistry of sulfide oxidation. American Chemical Society, Symposium Series, 550, 190-200

Nishimura, T., \& Robins, R. G. (2008). Confirmation that tooeleite is a ferric arsenite sulphate hydrate, and is relevant to arsenic stabilisation. Minerals Engineering, 21, 246-251.
Núñez-Olivera, E., Otero, S., Tomás, R., Fabón, G., \& Martínez-Abaigar, J. (2010). Cyclic environmental factors only partially explain the seasonal variability of photoprotection and physiology in two mosses from an unforested headwater stream. The Bryologist, 113(2), 277-291.

Pentacost, A. (2005). Travertine. Berlin/Heidelberg: Springer.

Pentacost, A., \& Zhaohui, Z. (2002). Bryophytes from some travertine-depositing sites in France and the U.K.: relationships with climate and water chemistry. Journal of Bryology, 24(3), 233-241.

Ramírez, Á., \& Cano, A. (2005). Líquenes de Pueblo Libre, una localidad andina en la Cordillera Negra (Huaylas, Ancash, Perú). lichens of Pueblo Libra, an Andean locality from Cordillera Negra (Huaylas, Ancash, Peru). Revista Peruana de Biología, 12(3), 383-396.

Salvador, F., Monerris, J., Rochefort, L. (2014/15): Peatlands of the Peruvian Puna ecoregion: types, characteristics and disturbance. Mires and Peat, 15, 1-17.

Samecka-Cymerman, A., Kolon, K., \& Kempers, A. J. (2002). Heavy metals in aquatic bryophytes from the Ore mountains (Germany). Ecotoxicology and Environmental Safety, 52, 203-210.

Sand-Jensen, K., Riis, T., Markager, S., \& Vincent, W. F. (1999). Slow growth and decomposition of mosses in Arctic lakes. Canadian Journal of Fisheries and Aquatic Sciences, 56, 388-93.

Schwarz, A. M. J., Green, T. G. A., \& Seppelt, R. D. (1992). Terrestrial vegetation at Canada Glacier, Southern Victoria Land, Antarctica. Polar Biology, 12, 397-404.

Schwertmann, U., \& Cornell, R. M. (2000). Iron oxides in the laboratory: preparation and characterization. Weinheim: Wiley-VCH Verlag.

Selkirk, P. M., \& Skotnicki, M. L. (2007). Measurement of moss growth in continental Antarctica. Polar Biology, 30, 407-413.

Shacklette, H. T. (1965). A leafy liverwort hydrosere on Yakobi Island, Alaska. Ecology, 46, 377-378.

Shaw, J. (1987). Evolution of heavy metal tolerance in bryophytes. II. an ecological and experimental investigation of the "copper moss," scopelophila cataractae (Pottiaceae). American Journal of Botany, 74(6), 813-821.

Spence, J. R., \& Ramsey, H. P. (2002). The genus Anomobryum Schimp. (Bryopsida, Bryaceae) in Australia. Telopea, 9, 777792.

Van Reeuwijk, L. P. (2002). Procedures for soil analyses. Technical report 9 (6th ed.). Wageningen: ISRIC.

Visual MINTEQ 3.0: a free equilibrium speciation model. KTH, Sweden. http://www2.1wr.kth.se. Accessed 25 November 2013.

Walsh, W. (2013). The origin and distribution of trace metals in the Rio Santa Watershed, Peru. MSc thesis. McGill University, Montreal, Quebec, Canada.

Waychunas, G. A., Xu, N., Fuller, C. C., Davis, J. A., \& Bigham, J. M. (1995). XAS study of $\mathrm{AsO} 4$ and $\mathrm{SeO} 4$ substituted schwertmannites. Physica B, 208(209), 481-483.

Yu, J. Y., Heo, B., Choi, I. K., Cho, J. P., \& Chang, H. W. (1999). Apparent solubilities of schwertmannite and ferrihydrite in natural stream waters polluted by mine drainage. Geochimica et Cosmochimica Acta, 63, 3407-3416.

Zander, R. H., \& Hegewald, E. (1976). Leptodontiella, gen. nov. and Leptodontium from Peru. The Bryologist, 79, 16-21. 